This is an Open Access article, distributed under the terms of the Creative Commons Attribution licence (http://creativecommons.org/licenses/by/4.0/), which permits unrestricted re-use, distribution, and reproduction in any medium, provided the original work is properly cited.

doi:10.1017/jfm.2018.546

\title{
Transition in a separation bubble under tonal and broadband acoustic excitation
}

\author{
John William Kurelek ${ }^{1,2}$, Marios Kotsonis ${ }^{2}$ and Serhiy Yarusevych ${ }^{1, \dagger}$ \\ ${ }^{1}$ Department of Mechanical and Mechatronics Engineering, University of Waterloo, N2L 3G1, \\ Waterloo, Canada \\ ${ }^{2}$ Department of Aerodynamics, Delft University of Technology, 2629HS, Delft, The Netherlands
}

(Received 30 October 2017; revised 4 June 2018; accepted 4 July 2018;

first published online 16 August 2018)

Transition and flow development in a separation bubble formed on an airfoil are studied experimentally. The effects of tonal and broadband acoustic excitation are considered since such acoustic emissions commonly result from airfoil self-noise and can influence flow development via a feedback loop. This interdependence is decoupled, and the problem is studied in a controlled manner through the use of an external acoustic source. The flow field is assessed using time-resolved, two-component particle image velocimetry, the results of which show that, for equivalent energy input levels, tonal and broadband excitation can produce equivalent changes in the mean separation bubble topology. These changes in topology result from the influence of excitation on transition and the subsequent development of coherent structures in the bubble. Both tonal and broadband excitation lead to earlier shear layer roll-up and thus reduce the bubble size and advance mean reattachment upstream, while the shed vortices tend to persist farther downstream of mean reattachment in the case of tonal excitation. Through a cross-examination of linear stability theory (LST) predictions and measured disturbance characteristics, nonlinear disturbance interactions are shown to play a crucial role in the transition process, leading to significantly different disturbance development for the tonal and broadband excited flows. Specifically, tonal excitation results in transition being dominated by the excited mode, which grows in strong accordance with linear theory and damps the growth of all other disturbances. On the other hand, disturbance amplitudes are more moderate for the natural and broadband excited flows, and so all unstable disturbances initially grow in accordance with LST. For all cases, a rapid redistribution of perturbation energy to a broad range of frequencies follows, with the phenomenon occurring earliest for the broadband excitation case. By taking nonlinear effects into consideration, important ramifications are made clear in regards to comparing LST predictions and experimental or numerical results, thus explaining the trends reported in recent investigations. These findings offer new insights into the influence of tonal and broadband noise emissions, resulting from airfoil self-noise or otherwise, on transition and flow development within a separation bubble.

Key words: aeroacoustics, boundary layer separation, transition to turbulence

$†$ Email address for correspondence: syarus@uwaterloo.ca 


\section{Introduction}

Flow separation on lifting surfaces operating at low Reynolds numbers, $R e \lesssim 5 \times 10^{5}$, is common due to the inherently poor ability of laminar boundary layers to endure adverse pressure gradients, encountered primarily on the suction side of lift-generating surfaces. Common applications operating in this Reynolds number domain include gliders, unmanned aerial vehicles and small-to-medium scale turbo-machinery (Mueller \& DeLaurier 2003; Hodson \& Howell 2005), where flow separation typically leads to undesirable effects, including loss of lift, increase in drag, unsteady loading and/or noise emissions (Carmichael 1981; Arcondoulis et al. 2010). Airfoil self-noise emissions can manifest in this flow regime (Brooks, Pope \& Marcolini 1989) and can either be tonal or broadband (e.g. Amiet 1976; Brooks \& Hodgson 1981; Nash, Lowson \& McAlpine 1999; Pröbsting, Serpieri \& Scarano 2014). While characteristics of the acoustic perturbations depend significantly on boundary layer development over the airfoil, the resulting noise emissions can influence flow development via a feedback loop (e.g. Pröbsting \& Yarusevych 2015). The present work is therefore focused on evaluating the effect of tonal and broadband acoustic forcing on flow over an airfoil operating at a low Reynolds number. The inherent complexity of the aeroacoustic problem is simplified by providing acoustic forcing from a controlled source, allowing for a detailed evaluation of the induced effect on the flow field.

A typical progression of the flow development over the suction side of a lifting surface operating in the low Reynolds number regime is illustrated in figure 1. A laminar separated shear layer forms as a result of boundary layer separation and undergoes transition to turbulence. Depending on flow conditions and geometry of the surface, shear layer transition can lead to flow reattachment in the mean sense, forming a closed region of recirculating fluid referred to as a laminar separation bubble (LSB). The development of LSBs on airfoils, as well as flat plates subjected to adverse pressure gradients, have been examined in a number of previous investigations, with recent detailed literature reviews found in Marxen \& Henningson (2011) and Boutilier \& Yarusevych (2012c). The transition process within the separated shear layer involves selective amplification of small-amplitude disturbances developing upstream of the separation point, which serve as the initial condition for the instabilities within the LSB (Diwan \& Ramesh 2009; Michelis, Kotsonis \& Yarusevych 2018). Within the fore portion of the bubble, the initial stage of transition has been found to be well modelled by LST and the primary amplification of perturbations is attributed to an inviscid Kelvin-Helmholtz instability (Häggmark, Hildings \& Henningson 2001; Rist \& Maucher 2002; Marxen et al. 2003; Boutilier \& Yarusevych 2012c). However, an absolute instability can also manifest in LSBs when reverse flow velocities reach approximately 15-20\% of the free-stream velocity (e.g. Alam \& Sandham 2000; Rist \& Maucher 2002). This has been explored recently using global stability analysis by Rodríguez \& Theofilis (2010) and Rodríguez, Gennaro \& Juniper (2013), showing that stationary three-dimensional instability modes can be observed at lower reverse flow velocities (e.g. $7 \%$ of the free-stream velocity).

The growth of unstable disturbances in the shear layer eventually leads to shear layer roll-up in the aft portion of the bubble (figure 1), where quasi-periodic vortex shedding occurs (e.g. Watmuff 1999; Lang, Rist \& Wagner 2004; Yarusevych, Sullivan \& Kawall 2009; Serna \& Lázaro 2014). The formed structures dominate the flow development in the aft portion of the bubble (Lengani et al. 2014, 2017) and have been argued to be responsible for inducing mean reattachment (e.g. Marxen \& Henningson 2011; Yarusevych \& Kotsonis 2017a). At roll-up, the shear layer vortices 


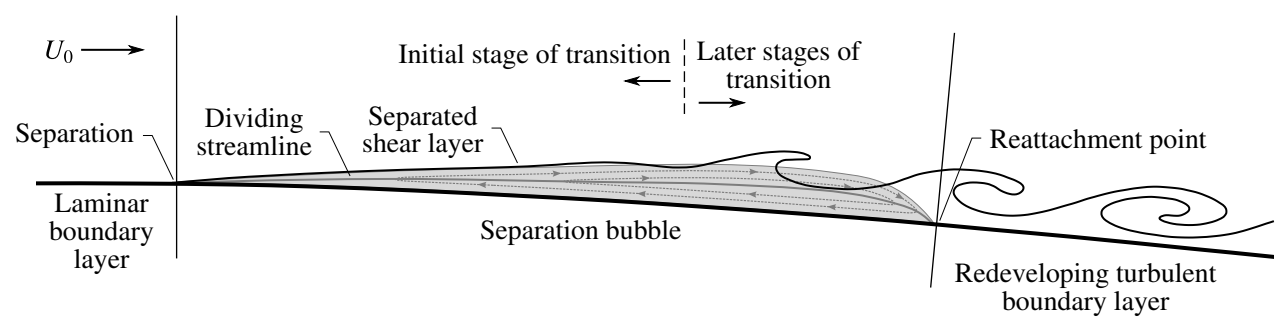

FIGURE 1. Time-averaged (grey lines) and unsteady features of a separation bubble.

have been found to be largely spanwise uniform, but quickly undergo significant three-dimensional deformations prior to the breakdown to turbulence (Jones, Sandberg \& Sandham 2008; Marxen, Lang \& Rist 2013; Nati et al. 2015; Kurelek, Lambert \& Yarusevych 2016; Kirk \& Yarusevych 2017). In contrast, other investigators have reported highly deformed and spanwise non-uniform vortical structures at formation (Burgmann \& Schröder 2008; Hain, Kähler \& Radespiel 2009; Wolf et al. 2011). Michelis et al. (2018) have recently proposed a theory that unifies these disparate descriptions, as they note that the degree to which deformations occur in the vortex filaments depends on the relative amplitude between the primary Kelvin-Helmholtz perturbation and any oblique modes which may be active in the flow upstream of separation.

The discussed aspects of separated shear layer development and laminar-to-turbulent transition are also directly related to airfoil self-noise, in particular, tonal and broadband noise generated at the trailing edge (e.g. Brooks et al. 1989). Strong acoustic tones are commonly observed on airfoils operating in the low Reynolds number domain (Paterson et al. 1973; Nash et al. 1999; Pröbsting et al. 2014). This occurs when laminar boundary layer separation or LSB formation occurs close to the trailing edge, leading to the strongly coherent perturbations amplified in the separated shear layer producing tones when scattered at the airfoil trailing edge (e.g. Arbey \& Bataille 1983; Desquesnes, Terracol \& Sagaut 2007; Pröbsting, Scarano \& Morris 2015). In contrast, when the LSB is located closer to the leading edge and a turbulent boundary layer forms over the aft portion of the airfoil, broadband noise emissions are produced at the trailing edge (Amiet 1976; Brooks \& Hodgson 1981).

The occurrence of such noise emissions is expected to have notable effect on the flow development over an airfoil, as laminar-to-turbulent transition is inherently sensitive to various free-stream disturbances. Indeed, in aeroacoustic investigations, the upstream propagation of tonal noise has been shown to establish an acoustic feedback loop (Arbey \& Bataille 1983; Atassi 1984; Jones \& Sandberg 2011; Plogmann, Herrig \& Würz 2013), thus affecting the development of shear layer perturbations responsible for the noise emissions. Pröbsting \& Yarusevych (2015) demonstrate that such a feedback loop, dictated by either suction or pressure side events, can alter LSB characteristics. In fact, many previous investigations have used controlled periodic forcing to gain insight into transition within LSBs. This type of forcing has been introduced in experimental and numerical studies by various means, including wall oscillations (Alam \& Sandham 2000; Lang et al. 2004; Marxen \& Rist 2010), external acoustic excitation (Jones, Sandberg \& Sandham 2010) and wall-mounted plasma actuators (Yarusevych \& Kotsonis 2017a,b). The sensitivity of the LSB to periodic forcing has also been explored for flow control purposes aimed at stall control and improvement of airfoil performance at pre-stall conditions, for example, 
using synthetic jets (Amitay \& Glezer 2002; Glezer, Amitay \& Honohan 2005) and external acoustic excitation (Zaman, Bar-Sever \& Mangalam 1987; Nishioka, Asai \& Yoshida 1990; Yarusevych, Sullivan \& Kawall 2007).

Several investigators have linked the optimal excitation frequency to the frequency of the most amplified disturbances in the natural flow (Yarusevych et al. 2007; Marxen et al. 2015; Yarusevych \& Kotsonis 2017b), showing that inducing flow reattachment on a stalled airfoil through the formation of an LSB or reducing the size of an existing LSB is most effective when the excitation frequency targets the Kelvin-Helmholtz instability and the associated vortex shedding process. Yarusevych \& Kotsonis (2017a) demonstrate that forcing at this 'fundamental' frequency has a significant impact on the ensuing vortex dynamics, as the shedding process locks on to the excitation frequency and the coherence of the structures is increased. They related this to a higher entrainment of momentum from the outer flow to the surface and thus an upstream advancement in the mean reattachment location. Increases to the excitation amplitude at these conditions lead to further upstream advances in the vortex roll-up and mean reattachment locations and thus further reductions in the LSB size. Such changes in the mean flow, however, lead to changes in flow stability characteristics, which Marxen \& Henningson (2011) and Marxen et al. (2015) refer to as the mean flow deformation. They note that, as the bubble size is reduced with increasing input perturbation amplitude, the spatial growth rates and frequency of most amplified disturbances are reduced. These effects on stability have since been experimentally supported and quantified by Yarusevych \& Kotsonis (2017b).

The foregoing discussion indicates that airfoil self-noise emissions, either tonal or broadband, are expected to influence flow over an airfoil, in general, and LSB characteristics, in particular. However, the inherent coupling between these two phenomena makes it difficult to assess the effect of airfoil self-noise emissions on LSB development. Furthermore, a cross-examination between the effects of tonal and broadband noise emissions on LSB dynamics has yet to be done in a controlled environment. Such an investigation has merit since the transition process within a laminar separation bubble is broadband in nature (e.g. Boiko et al. 2002), i.e. the flow is unstable to disturbances over a range of frequencies. Thus, the nonlinear mechanisms by which amplified perturbations interact in separation bubble flows are hypothesized to have significant impacts on flow development, since such mechanisms play key roles in the later stages of the transition processes in other canonical flows. In the case of the laminar boundary layer, the growth and interaction of instability modes leads to nonlinear and triadic resonance mechanisms (Craik 1971; Herbert 1988) which have been experimentally linked to the generation of coherent structures and the cascade to turbulence (Klebanoff, Tidstrom \& Sargent 1962; Kachanov \& Levchenko 1984; Saric \& Thomas 1984). Furthermore, Boiko et al. (2002) note that the interaction of waves with similar but slightly detuned frequencies are important. In evaluating the results of Kachanov, Kozlov \& Levchenko (1982), they note that the continued growth of two Tollmien-Schlichting (TS) waves with close but distinct frequencies incites the growth of fluctuations at not only their own frequencies and harmonics, but also at the sum and difference frequencies and their harmonics. All of these waves grow, interact and generate further harmonics, thus quickly realizing a broadband spectra of fluctuations. Similar observations have been made in investigations of free shear layers excited at multiple frequencies (Miksad 1972, 1973; Wygnanksi \& Petersen 1987; Mankbadi 1991; Raman \& Rice 1991). By exciting two distinct frequencies acoustically, Miksad (1973) found the same generation of fluctuations at sum and difference frequencies, in addition to harmonics 
and subharmonics. Moreover, nonlinear mode competition between disturbances was found to be important, as the two excited modes suppressed each other's growth, with a tendency for fluctuating energy to become distributed among a broad range of frequencies. Thus, it was necessary to employ a disturbance growth model that took nonlinear effects into account to accurately describe the transition process. A simple empirical-based Landau type model (Stuart 1962; Landau \& Lifschitz 1987) was shown to perform adequately, while more sophisticated methods, such as the nonlinear extension of the parabolized stability equations (Herbert 1997), have emerged since.

The present investigation is carried out to determine the effects of tonal and broadband acoustic excitation on flow development and transition in a laminar separation bubble. Disturbances are introduced in a controlled manner from an external source, so as to decouple the interdependence of flow development and acoustic emissions due to airfoil self-noise production. In addition to the unperturbed case, where broadband disturbances present in the environment serve as input to the unstable flow, two excitation cases are considered: (i) tonal excitation at the frequency of the most amplified disturbances in the natural flow, and (ii) broadband excitation: white noise band-pass filtered to the unstable frequency range of the natural flow. The aim is then to cross-examine these cases and elucidate any underlying physical differences in the transition processes and the associated changes in flow development. The flow field is assessed via time-resolved, two-component particle image velocimetry (PIV). Two separate experimental configurations are employed to characterize streamwise and spanwise aspects of the flow development. The time-resolved nature of the measurements allows for characterization across the entire range of relevant time scales. The experimental results are complemented by LST analysis, and the elucidated differences in the evolution of perturbations are related to changes in vortex dynamics and mean topological features of the bubble.

\section{Experimental set-up}

Experiments were conducted in the closed-loop wind tunnel located at the University of Waterloo's Fluid Mechanics Research Laboratory. The tunnel test section is $0.61 \times 0.61 \mathrm{~m}$ in cross-section, $2.44 \mathrm{~m}$ long and features full optical access. The tunnel has a 9:1 contraction ratio, upstream of which the flow is conditioned by a honeycomb insert and a set of five screens, resulting in a free-stream turbulence intensity less than $0.1 \%$ and an integral length scale of $0.2 c$, where $c$ is the airfoil chord length, with velocity signals low-pass filtered at $10 \mathrm{kHz}(S t=208)$. Here, the Strouhal number is defined as $S t=f c / U_{0}$, where $f$ and $U_{0}$ denote frequency and the free-stream velocity, respectively. Furthermore, the free-stream velocity was verified to have no significant spectral content within the frequency range of interest to this investigation, $100 \leqslant f \leqslant 2000 \mathrm{~Hz}(2 \leqslant S t \leqslant 42)$. The test section free-stream uniformity was verified to be within $\pm 0.5 \%$. The free-stream velocity was set based on the contraction static pressure drop calibrated against a Pitot-static tube in the empty test section, with the associated uncertainty in the free-stream velocity estimated to be less than $2 \%$.

Tests were performed using an aluminium NACA 0018 airfoil model with a chord length and span of 0.2 and $0.61 \mathrm{~m}$, respectively. A diagram of the model is shown in figure 2, where the surface-attached coordinate system $(x, y, z)$ is defined. The model has 95 static pressure taps of $0.4 \mathrm{~mm}$ diameter distributed over the suction and pressure surfaces, 65 of which are located at the mid-span plane. Mean surface 


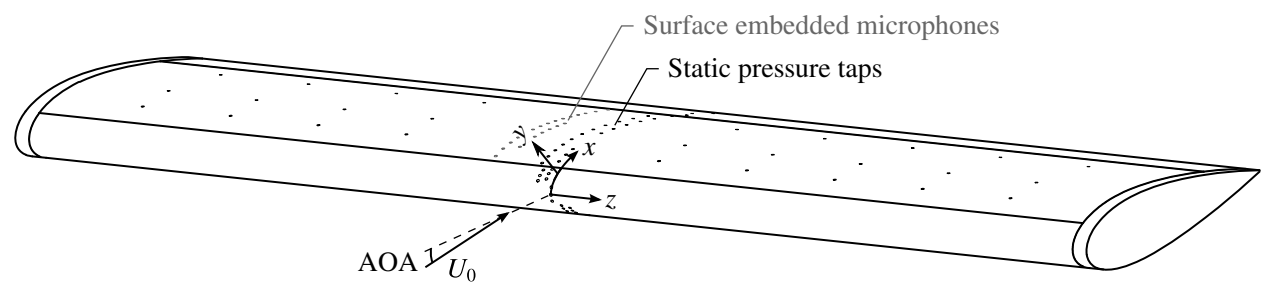

FIgURE 2. Airfoil model. Surface-attached coordinate system shown, with $x$ and $z$ measured along the surface from the leading edge and mid-span, and $y$ measured normal to the surface.

pressure measurements were conducted by multiplexing the pressure taps through a mechanical Scanivalve unit to Setra 239 differential pressure transducers, whose full range is $500 \mathrm{~Pa}$. The measurements were taken relative to the free-stream static pressure, measured via a pressure tap installed in the test section wall two chord lengths upstream of the airfoil leading edge. The uncertainty in the mean surface pressure measurements is estimated to be $2 \%$ of the free-stream dynamic pressure. The model is also equipped with 25 Panasonic WM-62C back electret condenser microphones installed under $0.8 \mathrm{~mm}$ diameter ports, twenty two of which are distributed along the chord in a staggered row located at $z / c \approx-0.2$, as shown in figure 2. Each microphone was calibrated in the airfoil model relative to a reference 4189 Brüel and Kjær microphone. All microphones have a flat response $( \pm 1 \mathrm{~dB})$ in the range $100 \leqslant f \leqslant 2000 \mathrm{~Hz}(2 \leqslant S t \leqslant 42)$. For all acoustic measurements, up to eight microphones were sampled simultaneously at $40 \mathrm{kHz}$ for a total of $2^{22}$ samples using a National Instruments PCI-4472 data acquisition card, which applies a hardware low-pass filter at the Nyquist limit to all sampled signals.

All tests were performed at an angle of attack (AOA) of $4^{\circ}$ and a free-stream velocity of $9.6 \mathrm{~m} \mathrm{~s}^{-1}$, corresponding to a chord-based Reynolds number of $R e=$ $U_{0} c / v=125000$. The solid blockage ratio at the investigated angle of attack is $6.1 \%$, and no blockage corrections were applied to the measured surface pressure distributions (Boutilier \& Yarusevych 2012a). The angle of attack was set using a digital protractor with a resolution of $0.1^{\circ}$. For the given flow conditions, separation bubbles form on both the suction and pressure surfaces, with the latter forming close to the trailing edge. To avoid the establishment of a feedback loop between the suction side transition process and tonal noise emissions due to the pressure side LSB (Pröbsting \& Yarusevych 2015), the pressure side boundary layer was tripped at $x / c=0.4$ by a $10 \mathrm{~mm}$ wide strip of randomly distributed three-dimensional roughness elements. The effectiveness of the trip in suppressing tonal noise due to pressure side separation was verified via microphone measurements.

Time-resolved, two-component PIV was employed in two separate planes to characterize spatio-temporal flow development in the separation bubble and the effect of acoustic excitation. The tests were performed in the two configurations depicted in figure 3. The flow was seeded using a glycol-water-based fog with a mean particle diameter of the order of $1 \mu \mathrm{m}$, whose characteristic response frequency (Melling 1997; Raffel et al. 2007) is above the Nyquist limit of the PIV sampling frequencies. The flow was illuminated by a laser sheet produced by a Photonics DM20-527 high-repetition rate $\mathrm{Nd}$ :YLF pulsed laser. The laser beam was introduced through the side wall of the test section and conditioned into a sheet approximately $1 \mathrm{~mm}$ 
(a)

(b)
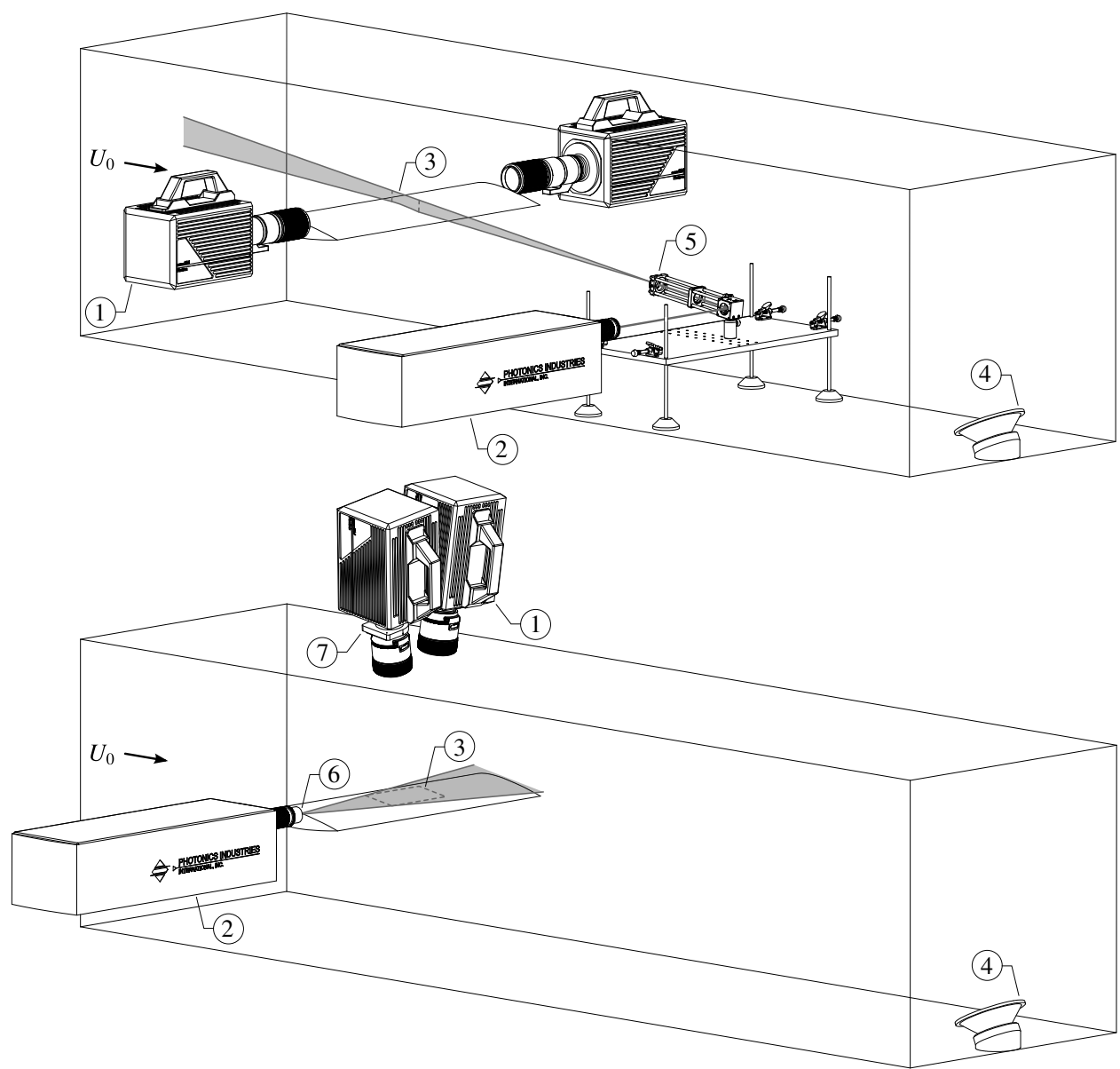

FIGURE 3. (a) Side-view and (b) top-view experimental configurations for PIV measurements. (1) High-speed cameras; (2) laser with focusing optics; (3) field of view; (4) speaker; (5) side view sheet optics located $3 c$ downstream of airfoil trailing edge; (6) top view sheet optics; (7) Scheimpflug adapter.

thick. For the side-view configuration (figure $3 a$ ), the sheet was oriented parallel to the airfoil cross-section and passed through the mid-span plane. Introducing the laser sheet tangent to the airfoil surface effectively mitigated surface reflections. For the top view (figure $3 b$ ), the sheet was oriented parallel to the airfoil surface within the investigated field of view (FOV). For both configurations, images were captured by two Photron SA4 high-speed cameras synchronized with the laser via a LaVision timing unit controlled through LaVision's DaVis 8 software.

Table 1 provides an overview of the parameters for the PIV experiments. For the side-view configuration (figure $3 a$ ), the high-speed cameras were fitted with Nikon $200 \mathrm{~mm}$ fixed focal length macro lenses set to an aperture number $\left(f_{\#}\right)$ of 4 . The cameras' sensors were cropped to $1024 \times 512$ px and the fields of view were adjusted to maximize the spatial resolution in the aft portion of the separation bubble, while maintaining equal magnification factors of 0.67 for both cameras. The fields of view were overlapped by $10 \%$ and images were acquired in double-frame mode at 


\begin{tabular}{lccc} 
Parameter & $\begin{array}{c}\text { Side view } \\
\text { Photonics DM20-527 }\end{array}$ & Top view & Unit \\
Laser & \multicolumn{2}{c}{ Photron SA4 } \\
Cameras & 200 & 105 & $\mathrm{~mm}$ \\
Lens focal length & 4 & 2.8 & \\
Lens $f_{\#}$ & 0.67 & 0.33 & \\
Magnification factor & $1024 \times 512$ & $1024 \times 1024$ & $\mathrm{px}$ \\
Sensor resolution & $54 \times 12.5$ & $54 \times 102$ & $\mathrm{~mm}$ \\
Total field of view & \multicolumn{2}{c}{ Double-frame } & \\
PIV mode & 3.8 & 1.95 & $\mathrm{kHz}$ \\
Sampling rate & 40 & 60 & $\mu \mathrm{s}$ \\
Frame separation time & 17 & 9 & $\mathrm{px}$ \\
Outer flow displacement & 5456 & 2728 & \\
Number of samples & \multicolumn{2}{c}{$16 \times 16$} & $\mathrm{px}$ \\
Window size & 0.12 & 0.24 & $\mathrm{~mm}$ \\
Vector pitch & \multicolumn{4}{c}{}
\end{tabular}

TABLE 1. PIV parameters.

a sampling rate of $3.8 \mathrm{kHz}$. For the top-view PIV measurements (figure $3 b$ ), the cameras were fitted with Nikon $105 \mathrm{~mm}$ fixed focal length macro lenses set to $f_{\#}=2.8$. The streamwise extent of the FOV was set to match that of the combined FOV for the side-view configuration. The second camera was employed to extend the FOV in the spanwise direction, while maintaining an overlap of $10 \%$. For both cameras, the full sensor resolution of $1024 \times 1024 \mathrm{px}$ and equal magnification factors of 0.33 were used. Images were acquired in double-frame mode at a rate of $1.95 \mathrm{kHz}$. In this configuration, one camera was set normal to the field of view and the second camera had to be tilted (figure $3 b$ ). Thus, to maintain the entire field of view in focus, the second camera was equipped with a Scheimpflug adapter.

For both PIV configurations, the focus was adjusted to produce particle images approximately 2-3 px in diameter. An iterative multi-grid cross-correlation scheme with window deformation (Scarano \& Riethmuller 2000) was used to compute velocity fields. A final interrogation window size of $16 \times 16$ px with $75 \%$ overlap was selected, with each window containing, on average, 14 particles. As a result, the vector pitches in the PIV data are $0.12 \mathrm{~mm}$ and $0.24 \mathrm{~mm}$ for the side and top-view configurations, respectively. The results were post-processed using the universal outlier detection algorithm (Westerweel \& Scarano 2005). Once the respective vector fields were calculated, the mean velocity fields for each camera were cross-correlated in the overlap region to align the FOVs. The vector fields were then interpolated onto the surface-attached coordinate system with a cosine weighted blending function employed in the overlap region. The random errors in the PIV measurements were evaluated using the correlation statistics method (Wieneke 2015). The associated average uncertainties within the region of the separated shear layer are estimated to be less than $6 \%$ and $6.5 \%$ of $U_{0}$ for the side and top-view configurations, respectively, while higher uncertainties (approximately $10 \%$ of $U_{0}$ ) are present near the wall. Comparable uncertainties for the two configurations were achieved by minimizing the out-of-plane loss of particles for the top view by selecting a shorter frame separation time (table 1). Uncertainty estimates for all critical quantities derived from the PIV measurements were obtained through uncertainty propagation (Moffat 1988).

Sound excitation was provided by a Pyramid WH88 sub-woofer placed within the test section on an vibration isolating pad, $6 c$ downstream of the airfoil trailing 
Case

Natural

Tone $(\mathrm{T})$

Broadband (B)
SPL $(\mathrm{dB})$

\section{9}

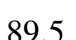

90.4

$92.7 \quad 94.9$

$\begin{array}{llll}89.5 & 90.4 & 92.7 & 94.9\end{array}$

TABLE 2. Investigated sound pressure levels. All SPLs calculated within $10.4 \leqslant S t \leqslant 20.8$.

edge. The presence of the speaker and the PIV sheet forming optics within the test section (figure 3) were confirmed via mean surface pressure measurements to have no measurable influence on mean LSB characteristics. The speaker was driven by an amplifier, with the excitation signal supplied by a National Instruments USB-6259 data acquisition unit. A 4189 Brüel and Kjær microphone was used to quantify the background noise level in the test section at $R e=125000$, as well as sound excitation at the airfoil suction surface in quiescent conditions. Based on the instrument's accuracy for a frequency range of $40-1000 \mathrm{~Hz}$, the uncertainty in the measured sound pressure levels (SPLs) is estimated to be $\pm 0.2 \mathrm{~dB}$. In addition, the amplitude of the sound excitation was verified to be uniform over the chordwise and spanwise extents of the investigated areas, as SPLs varied within $\pm 0.6 \mathrm{~dB}$.

In addition to the naturally developing flow, two types of excitation are investigated: (i) tonal excitation at the frequency of the most amplified disturbances in the unperturbed flow, i.e. the fundamental frequency, $S t_{0}=15.6$, and (ii) broadband: white noise band-pass filtered to the unstable frequency band of the unperturbed flow, $10.4 \leqslant S t \leqslant 20.8$. Spectra of fluctuating surface pressure near the natural separation point for all the investigated cases are presented in figure 4. For the natural case, a broad elevated energy content is seen in the spectrum around the fundamental frequency $\left(S t_{0}=15.6\right)$ and is attributed to natural transition occurring in the separated shear layer downstream of the measurement location. As expected for unforced transition in a low disturbance environment, the natural spectrum is devoid of any significant peaks, save for the relatively broadband acoustic activity centred at $S t=5.6$, which is due to acoustic standing waves that establish in all hard walled test sections (Parker 1966, 1967; Nash et al. 1999; Atobe, Tuinstra \& Takagi 2009). However, this activity is sufficiently removed from the fundamental frequency and the associated unstable band, thus ensuring negligible influence on the shear layer transition process. Figure 4 shows the spectra for the tonal and broadband excitation cases for the investigated and quiescent flow conditions. The results demonstrate that any given excitation is composed of only its intended frequencies and do not excite any resonant modes in the test facility. In order to facilitate proper comparison between tonal and broadband excitation cases, care was taken to ensure equivalent acoustic energy levels within the unstable frequency band of the natural flow, $10.4 \leqslant S t \leqslant 20.8$. A measure of such energy is the SPL for a given case computed within the unstable frequency band. For both excitation types considered, the same excitation amplitudes, in terms of SPL, were investigated: 89.5, 90.4, 97.2 and $94.9 \mathrm{~dB}$. The cases studied and their characteristic SPLs are summarized in table 2.

\section{Results}

The results presented herein pertain to experiments performed on a NACA 0018 airfoil at an angle of attack of $4^{\circ}$ and a chord Reynolds number of 125000 . For these conditions, the effects of tonal and broadband acoustic excitation on the transition process within a separation bubble are considered. 

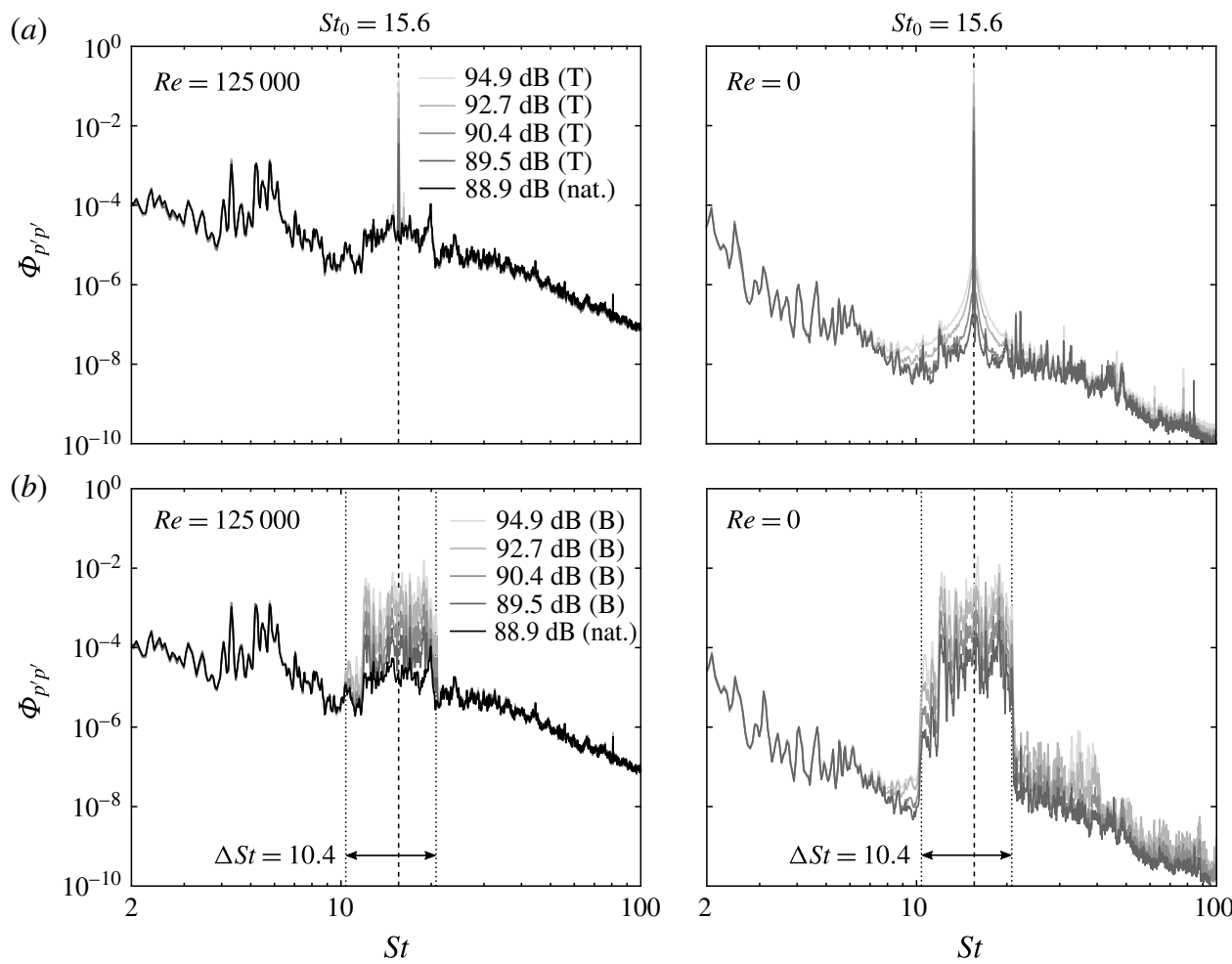

FIGURE 4. Spectra of fluctuating surface pressure $\left(\Phi_{p^{\prime} p^{\prime}}\right)$ measured near the natural separation point for $(a)$ tonal and $(b)$ broadband excitation in the investigated and quiescent flow conditions. St for quiescent condition computed using $U_{0}$ corresponding to $R e=125000$.

\subsection{Time-averaged flow field}

Mean surface pressure distributions are plotted in figure 5 and analysed to identify the presence and extent of the separation bubble. Surface pressure is presented in terms of a pressure coefficient, $C_{P}=\left(P-P_{0}\right) /\left[(1 / 2) \rho U_{0}^{2}\right]$, where $P_{0}$ and $\rho$ are the free-stream static pressure and density, respectively. For all cases, following the point of minimum pressure, the region of nearly constant pressure marks the presence of boundary layer separation (Tani 1964; O'Meara \& Mueller 1987). Using the methodology described by Boutilier \& Yarusevych (2012b) (cf. their figure 5), the mean separation $\left(x_{S}\right)$, transition $\left(x_{T}\right)$ and reattachment $\left(x_{R}\right)$ points can be estimated using the changes in slope in the pressure plateau and recovery regions, with the associated uncertainty dependent upon the spatial resolution of the pressure taps. Using this technique, the separation point is found to be $x_{S} / c=0.37 \pm 0.03$ for all cases. It is in the pressure recovery region, $0.46 \leqslant x / c \leqslant 0.68$, where the effect of excitation is most pronounced, as the introduction of disturbances and subsequent increase in excitation amplitude leads to a decreasing rate of pressure recovery within $0.53 \leqslant x / c \leqslant 0.68$. The results indicate that excitation likely causes the aft portion of the separation bubble to move upstream. In addition, the small yet discernible change in the slope of the pressure plateau within $0.37 \leqslant x / c \leqslant 0.48$ suggests that this may be accompanied by a delay in separation, which is less significant compared to the changes in the aft portion of the bubble. The associated variations in the estimated locations of mean 

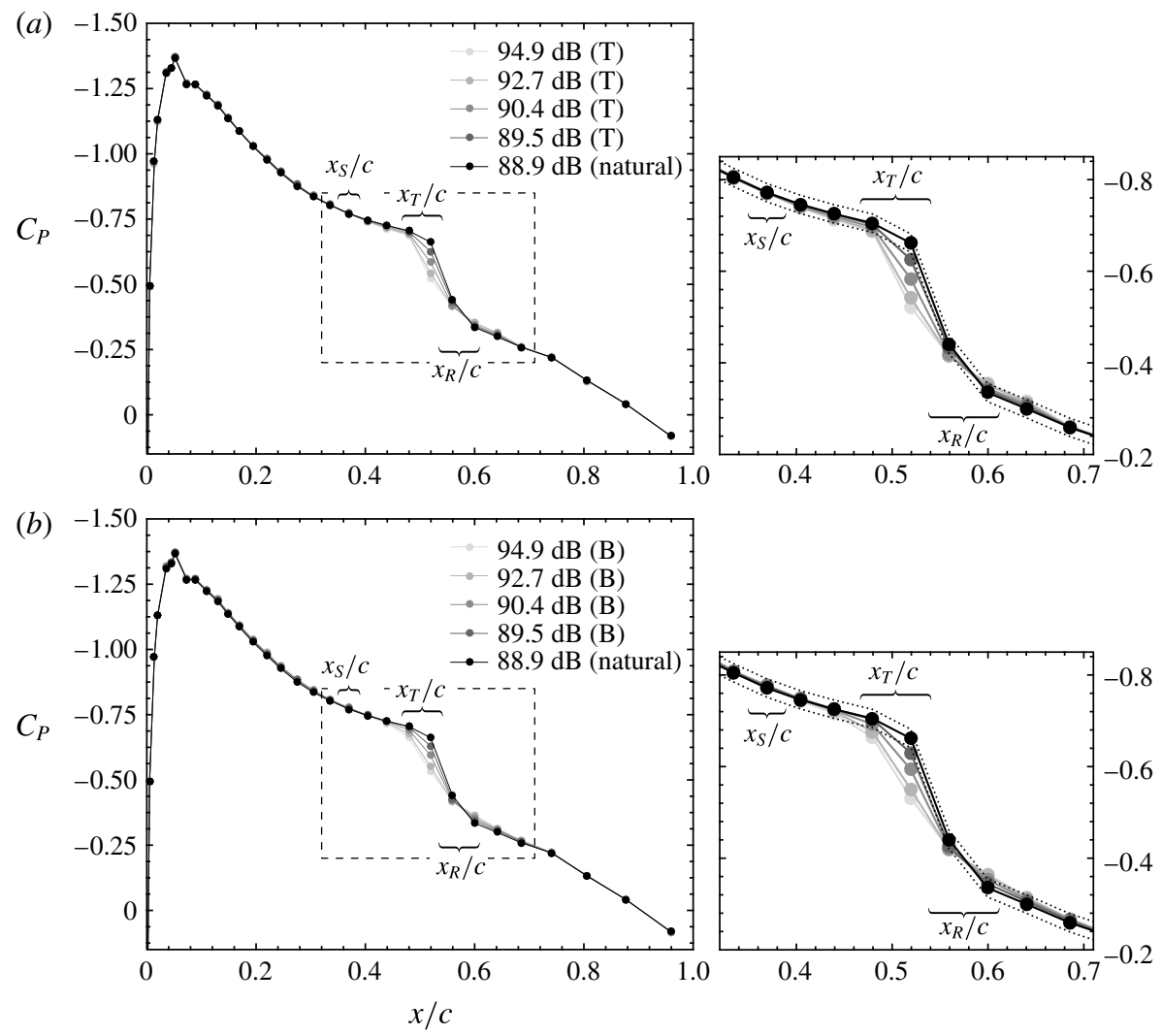

FIGURE 5. Effect of $(a)$ tonal and $(b)$ broadband excitation on mean suction surface pressure. Black dotted lines in magnified plots indicate uncertainty for the natural case.

separation, transition and reattachment fall within the spatial resolution limits of the measurements, and therefore cannot be quantified precisely based on these results.

Figure 6 depicts the effect of excitation of the time-averaged velocity field characteristics of the separation bubble. The mean outline of the separation bubble is identified using the locus of zero streamwise velocity points (Fitzgerald \& Mueller 1990), and is used to estimate the separation and reattachment points, in addition to the maximum bubble height $(h)$ and its streamwise location $\left(x_{h}\right)$. The uncertainties in determining $x_{S}, x_{h}$ and $x_{R}$ from the $U=0$ contour are indicated by the dotted lines in figure 6 , which are determined by propagating the PIV random error estimates and the uncertainty in locating the airfoil surface through the determination of these locations (Moffat 1988).

The mean streamwise velocity contours in figure 6 show the presence of a separation bubble that extends from $x_{S} / c=0.352 \pm 0.027$ to $x_{R} / c=0.565 \pm 0.009$ for the natural case, with the identified locations agreeing with the mean pressure distribution (figure 5). The bubble reaches its maximum height at $x_{h} / c=0.514 \pm 0.005$, which agrees with the onset of pressure recovery used to identify the mean transition location (figure 5). Reverse flow is present near the airfoil surface within the separation bubble, and the maximum reverse flow velocity across all cases examined is $4 \%$ of $U_{0}$, thus indicating the flow is only convectively unstable (Alam \& Sandham 2000; Rist \& Maucher 2002; Rodríguez \& Theofilis 2010; Rodríguez et al. 2013). 

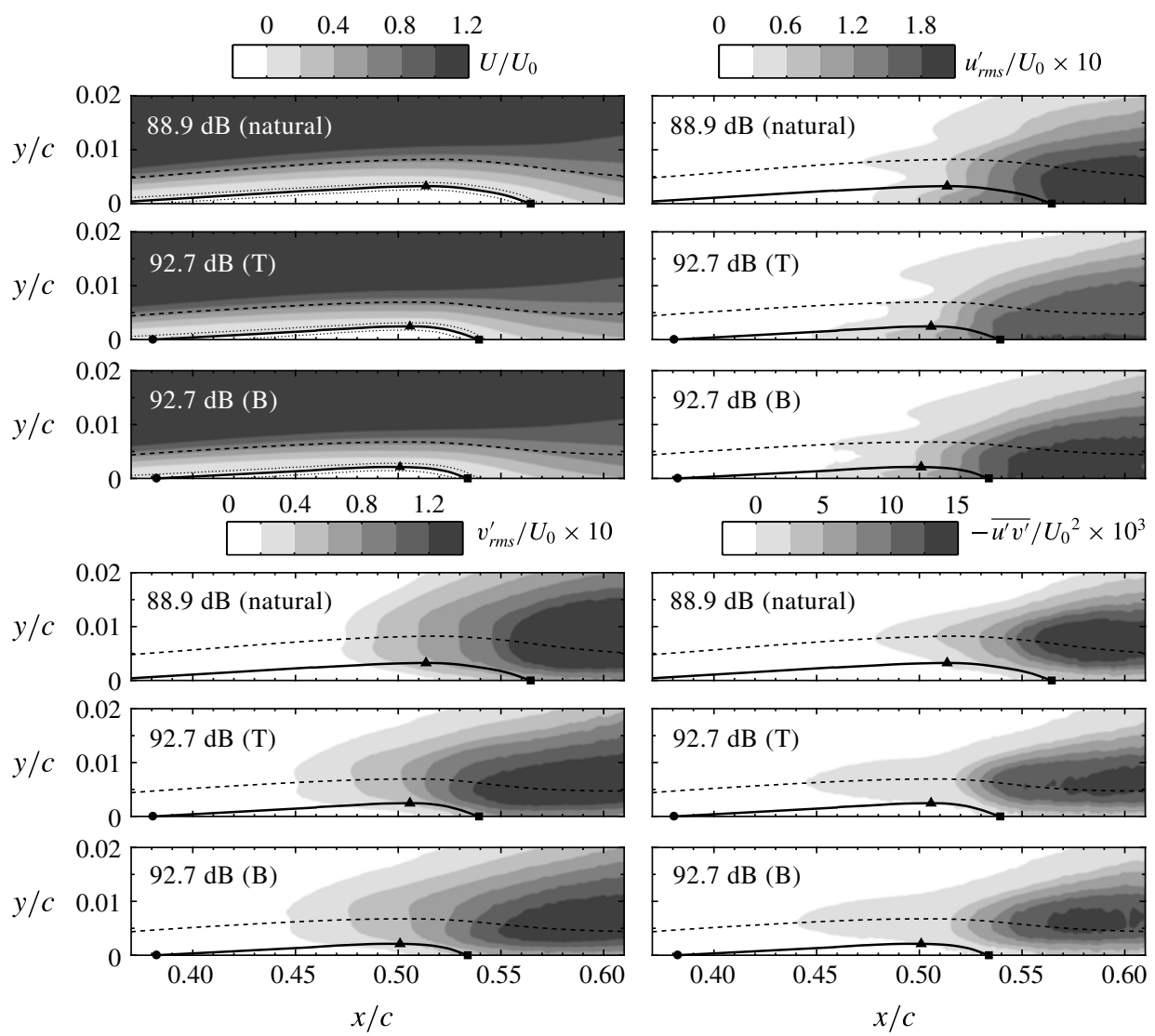

FIgURE 6. Mean $(U)$ and root-mean-square (r.m.s.) of fluctuating $\left(u_{r m s}^{\prime}, v_{r m s}^{\prime}\right)$ velocity contours, and Reynolds stress $\left(\overline{u^{\prime} v^{\prime}}\right)$ contours. Solid lines mark the $U=0$ contours, whose uncertainty limits are indicated by the dotted lines. Circle, triangle and square markers denote mean separation, maximum bubble height and reattachment points, respectively. Dashed lines indicate displacement thickness $\left(\delta^{*}\right)$.

In the presence of forcing, both tonal and broadband excitation cause the streamwise extent and height of the bubble to decrease. In particular, boundary layer separation is delayed, the maximum bubble height reduces and the mean reattachment point advances upstream, as has been reported for separation bubbles subjected to locally introduced periodic excitation (Marxen et al. 2015; Yarusevych \& Kotsonis 2017a,b). The changes in the separation point can be attributed to the mean flow deformation effect (Marxen et al. 2015), as changes to mean topology in the aft portion of the bubble affect the surface pressure distribution (figure 5), thereby affecting the separation location.

Integral shear layer parameters are computed from the PIV measurements, with normalization done with respect to the local edge velocity (i.e. mean velocity at local boundary layer thickness), and the top of the domain serving as the upper integration limit. These parameters are presented in figure 7, where, regardless of the excitation type, increases in excitation amplitude lead to reductions in the displacement thickness $\left(\delta^{*}\right)$. The momentum thickness $(\theta)$ does not change appreciably in the fore portion of the bubble, $x / c \lesssim 0.5$, where flow in the near-wall region is nearly stagnant. The 

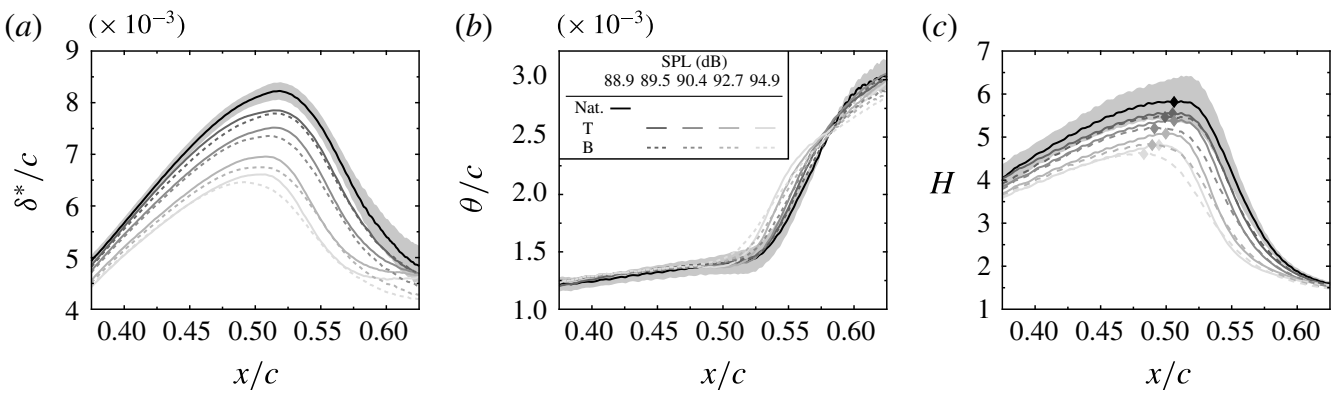

FIGURE 7. Effect of excitation on integral shear layer parameters: displacement thickness $\left(\delta^{*}\right)$, momentum thickness $(\theta)$ and shape factor $(H)$. Diamond markers denote shape factor maxima. Grey shaded regions denote uncertainty for the natural case.

onset of the rapid increase in the momentum thickness is advanced upstream when the excitation is applied at higher amplitudes. The observed increase in $\theta$ is due to the later stages of flow transition in the aft portion of the bubble, and it takes place where the growth in displacement thickness begins to saturate and, thus, the shape factor $\left(H=\delta^{*} / \theta\right)$ peaks. Shape factor maxima are indicated by the diamond markers in figure 7 , whose streamwise location is denoted by $x_{H}$. Good agreement is found between $x_{H}$ and the estimated locations of mean transition and maximum bubble height (figures 5 and 6, respectively). The observed trends are in agreement with previous reports of experimentally measured integral shear layer parameters in separation bubbles (Brendel \& Mueller 1988; Yarusevych \& Kotsonis 2017a).

The root-mean-square (r.m.s.) contour plots in figure 6 show the spatial amplification of velocity fluctuations in the separation bubble. In particular, the streamwise r.m.s. velocity field $\left(u_{r m s}^{\prime}\right)$ exhibits triple peak patterns for given wall-normal profiles, which are consistent with those reported in previous investigations (Watmuff 1999; Lang et al. 2004; Boutilier \& Yarusevych 2012c; Yarusevych \& Kotsonis 2017a). Upstream of mean reattachment, significant amplification follows the two near-wall peaks, indicating the growth of disturbances within the reverse flow region and the separated shear layer, with the latter following the displacement thickness. The strong amplification of wall-normal velocity fluctuations $\left(v_{r m s}^{\prime}\right)$ is also observed within the separated shear layer, with maximum values attained at the wall-normal location of the displacement thickness. In the presence of excitation, the r.m.s. contours reveal shear layer disturbances reach higher amplitudes at earlier streamwise locations; more clearly seen in the $v_{r m s}^{\prime}$ fields. Of particular interest is the amplitude of fluctuations reached at the bubble maximum height location. For the exemplary cases shown in figure 6 , and all other excitation amplitudes investigated, a relatively constant value of $u_{r m s}^{\prime}=v_{r m s}^{\prime} \approx 0.06 U_{0}$ is found at the maximum height location, regardless of excitation type or amplitude. The observation is noteworthy since this location is where pressure recovery begins, the momentum thickness begins to increase rapidly, and the $H$ factor reaches maximum values, indicating that time-averaged transition takes place when velocity fluctuations in the shear layer reach comparable critical amplitudes.

As expected, the streamwise development of the Reynolds shear stress $\left(\overline{u^{\prime} v^{\prime}}\right)$ is similar to that of the velocity fluctuations, with the locus of $\overline{u^{\prime} v^{\prime}}$ minima following the separated shear layer closely. Several investigators have relied on the Reynolds shear stress as an indicator of transition onset in the separated shear layer. Specifically, Ol et al. (2005) and Hain et al. (2009) identified $x_{T}$ as the point where $-\overline{u^{\prime} v^{\prime}}$ exceeded 


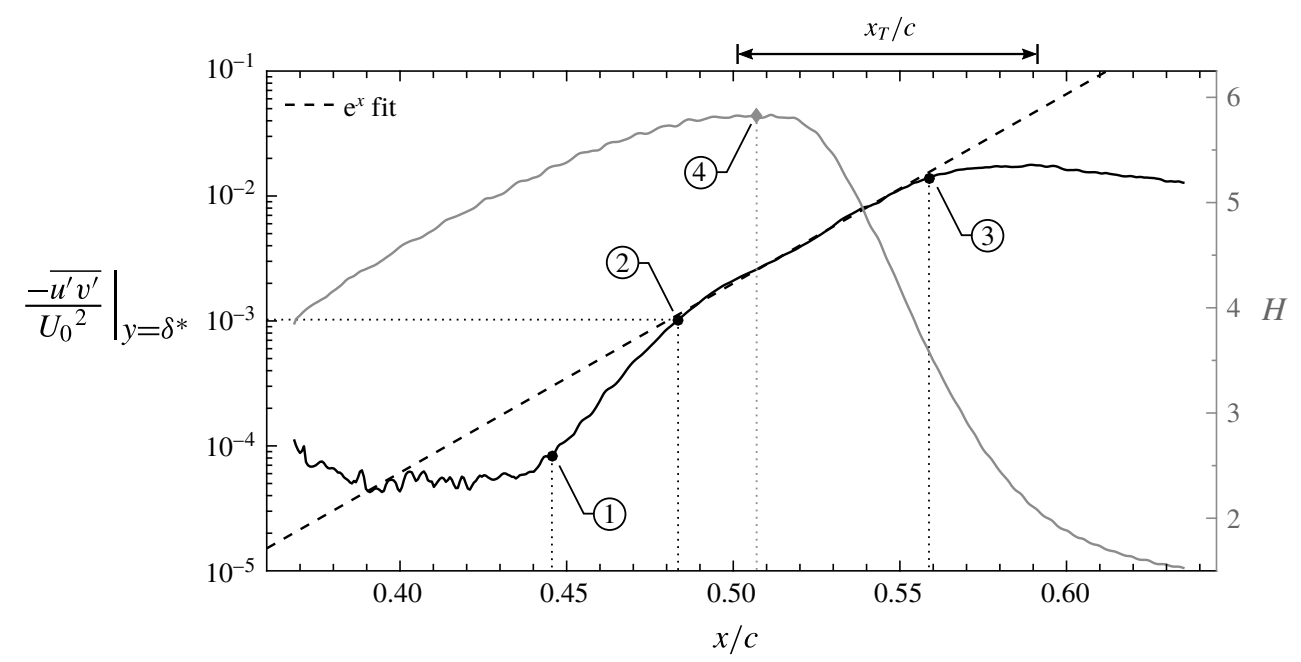

FIGURE 8. Comparison of techniques in estimating the natural mean transition point. (1) Sharp increase in Reynolds shear stress growth rate (Burgmann \& Schröder 2008); (2) threshold of $0.001 U_{0}^{2}$ (Ol et al. 2005; Hain et al. 2009); (3) deviation from exponential growth (Lang et al. 2004); (4) shape factor maximum (Brendel \& Mueller 1988; McAuliffe $\&$ Yaras 2005). Range for $x_{T}$ established from $C_{P}$ distribution (figure 5).

an arbitrary threshold of $0.001 U_{0}^{2}$, Burgmann \& Schröder (2008) used the points where the growth rate in $\overline{u^{\prime} v^{\prime}}$ sharply increased, and Lang et al. (2004) used the point where growth deviated from a fixed exponential rate. On the other hand, Brendel \& Mueller (1988) and McAuliffe \& Yaras (2005) used the streamwise maximum in shape factor as an estimate for transition onset. A comparison of these methods, contrasted with the estimate for $x_{T}$ from the surface pressure measurements, is presented in figure 8 . There is considerable variation in the results from the different estimation techniques, with the approaches of Ol et al. (2005) and Burgmann \& Schröder (2008) producing estimates that fall outside of the expected range, which can be attributed to the methods using an arbitrary threshold and being sensitive to the measurement noise level, respectively. The estimates from the shape factor method and that of Lang et al. (2004) agree with the pressure-based estimate, however the latter method is sensitive to the parameters used to determine the exponential curve fit and the amount by which the shear stress is allowed to deviate. Thus, utilizing the shape factor to identify a mean transition point is viewed as a more robust approach and is adopted in the present study.

The effects of excitation type and amplitude on the mean separation bubble characteristics are summarized in figure 9. Regardless of the type of excitation, increasing excitation amplitude leads to a continuous diminishment in the streamwise and wall-normal extents of the separation bubble, however, it should be noted that quantifying the exact changes in the separation location and maximum bubble height is difficult due to the relatively high uncertainties associated with these quantities. Upstream of $x_{H}$, i.e. in the fore portion of the bubble, disturbance amplitudes are relatively low (figure 6), and therefore their growth is expected to be well modelled by LST (e.g. Häggmark et al. 2001; Boutilier \& Yarusevych 2012c; Marxen et al. 2015). It can be conjectured that the upstream movement of the maximum shape factor is due to excitation providing higher initial disturbance amplitudes to which 

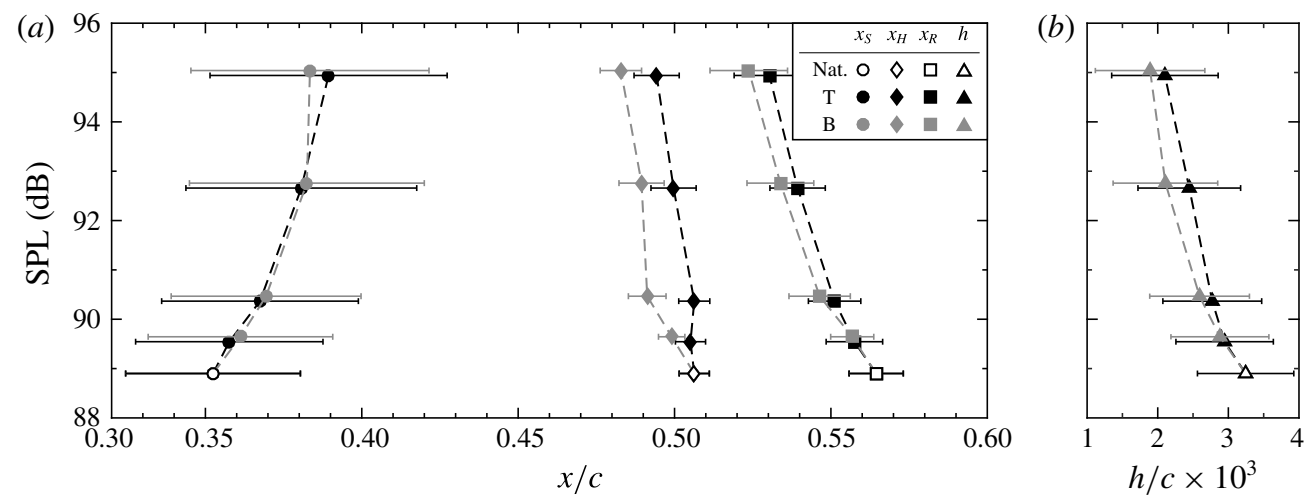

FIGURE 9. Effect of excitation on (a) mean streamwise locations of separation, maximum shape factor and reattachment, and $(b)$ maximum bubble height. Points of equal SPL are offset slightly in the vertical direction for clarity.

the LSB transition process is receptive. This assertion is examined in $\S 3.2$, where linear stability analysis is performed on the experimental data, in conjunction with an assessment of the effects of nonlinear interactions among disturbances. Such analysis sheds light on the differences between tonal and broadband excitation, as the results in figure 9 give a preliminary indication that broadband excitation can be as effective at accelerating transition as tonal excitation.

Excitation also reduces the size of the aft portion of the bubble, i.e. the region between $x_{H}$ and $x_{R}$. However, for all cases examined, the extent of the aft portion relative to the total bubble length, $\left(x_{R}-x_{H}\right) /\left(x_{R}-x_{S}\right)$, is nearly constant at approximately $25 \%$. Therefore both types of acoustic excitation are effective in proportionally decreasing both the fore and aft portion of the bubble. It is in the aft portion where shear layer roll-up occurs and the role of coherent structures is important (e.g. Marxen \& Henningson 2011; Kurelek et al. 2016; Yarusevych \& Kotsonis 2017b). How these phenomena are affected by the forcing is examined in detail in $\$ 3.3$.

\subsection{Growth and interaction of disturbances}

To study the convective streamwise amplification of disturbances, linear stability theory is employed, which provides a model for the amplification of small-amplitude disturbances in a parallel laminar flow (e.g. see Drazin \& Reid 1981; Schlichting \& Gersten 2000). The Orr-Sommerfeld equation governs disturbance growth:

$$
(U-\Omega / \alpha)\left(\frac{\mathrm{d}^{2} \widetilde{v}}{\mathrm{~d} y^{2}}-\alpha^{2} \widetilde{v}\right)-\frac{\mathrm{d}^{2} U}{\mathrm{~d} y^{2}} \widetilde{v}=-\frac{\mathrm{i} U_{e} \delta^{*}}{\alpha R e_{\delta^{*}}}\left(\frac{\mathrm{d}^{4} \widetilde{v}}{\mathrm{~d} y^{4}}-2 \alpha^{2} \frac{\mathrm{d}^{2} \widetilde{v}}{\mathrm{~d} y^{2}}+\alpha^{4} \widetilde{v}\right),
$$

where $\widetilde{v}$ is the mode of the wall-normal perturbation with angular frequency $\Omega$ and complex wavenumber $\alpha=\alpha_{r}+\mathrm{i} \alpha_{i}, U_{e}$ is the edge velocity, $\operatorname{Re}_{\delta^{*}}$ is the Reynolds number based on displacement thickness and edge velocity and $\mathrm{i}$ is the imaginary unit. A spatial formulation of the problem is employed (e.g. Schmid \& Henningson 2001), where $\Omega$ is prescribed and the eigenvalue problem is solved for $\alpha$, thus modelling the convective amplification of single frequency disturbances. Equation (3.1) is solved numerically using Chebyshev polynomial base functions and the companion 

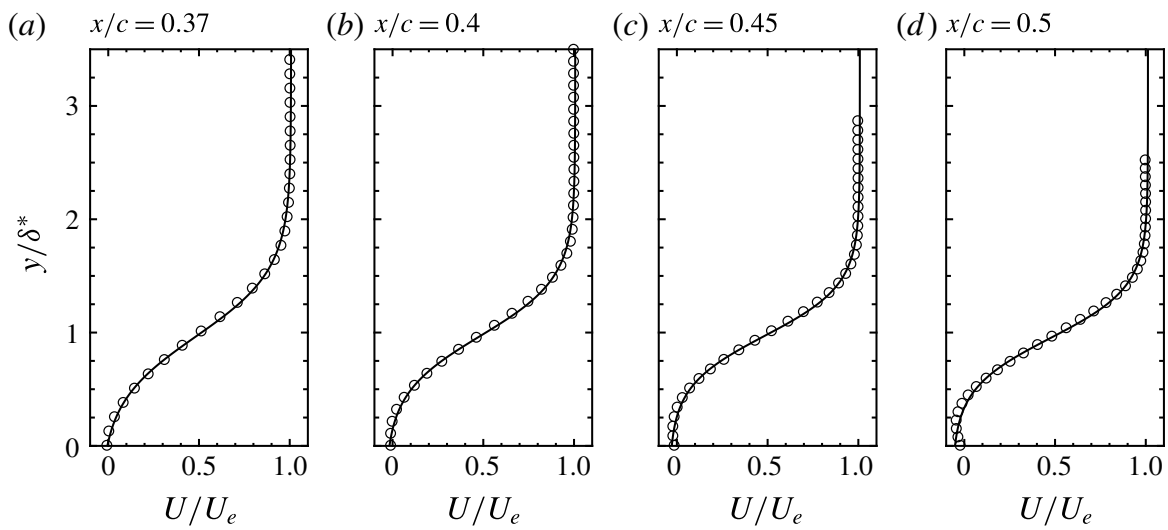

FIGURE 10. Measured velocity profiles (markers) in the natural flow and corresponding hyperbolic tangent fits (solid lines) used in LST calculations.

matrix technique to treat eigenvalue nonlinearity (Bridges \& Morris 1984). Additional information regarding the solution method can be found in van Ingen \& Kotsonis (2011).

Measured mean streamwise velocity profiles at given streamwise locations serve as input to the LST calculations, therefore making the analysis local. Spatial gradients estimated from PIV measurements often have a relatively high noise level due to the finite spatial resolution of the measurement technique (Westerweel, Elsinga \& Adrian 2013), to which LST predictions can be highly sensitive (Boutilier \& Yarusevych 2013). Therefore, stability analysis is performed using hyperbolic tangent fits to the experimental data, which have been shown to provide reasonable stability predictions (Dini, Selig \& Maughmer 1992; Boutilier \& Yarusevych 2012c) that are relatively insensitive to scatter in the data (Boutilier \& Yarusevych 2013). Exemplary velocity profiles and their corresponding fits for the natural flow conditions are shown in figure 10.

For validation purposes, results from the LST predictions and the experimental data are compared for the natural case in figure 11. A measure of amplitude growth is quantified from the LST results by integrating the spatial growth rates $\left(\alpha_{i}\right)$ in the downstream direction:

$$
A(x)=A_{0} \exp \left(\int_{x_{0}}^{x}-\alpha_{i} \mathrm{~d} x\right)
$$

where the integral term represents the amplification or $N$ factor. Here, $A$ is the disturbance amplitude, while $A_{0}$ and $x_{0}$ denote the amplitude and streamwise location at which the disturbance first becomes unstable, respectively. The location of $x_{0}$ is upstream of the PIV field of view and therefore cannot be determined directly, however, in the fore portion of the LSB, $\alpha_{i}$ may be approximated by a second-order polynomial (e.g. Jones et al. 2010, cf. figure 11). Based on this, $x_{0}$ can be estimated by extrapolating the curve fit to $\alpha_{i}=0$. In figure 11(a), the experimental spectrum of wall-normal velocity fluctuations shows an amplified band of disturbances within $10 \lesssim S t \lesssim 20$, with the highest energy content found approximately at $S t=15.6$, i.e. the fundamental frequency. The overlaid plot of $N$ factors shows good agreement between the LST predicted and experimental measured unstable frequency ranges, 

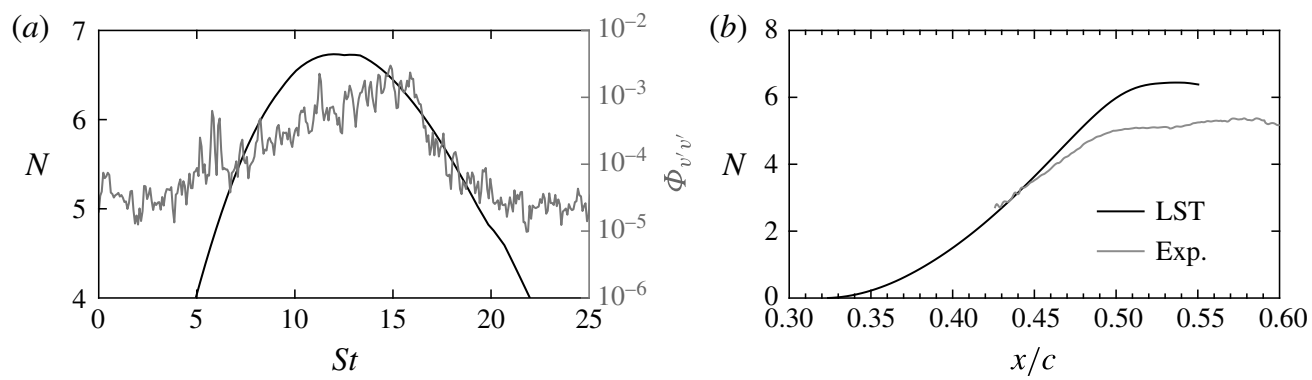

FIGURE 11. Validation of LST results for the natural flow. (a) LST $N$ factors and experimental spectrum of $v^{\prime}$ at the streamwise location of maximum bubble height. $(b)$ LST and experimental $N$ factors for $S t=15.6$.

with the most unstable frequency predictions differing by approximately $17 \%$. Such a discrepancy has been reported in similar studies (Yarusevych, Kawall \& Sullivan 2008; Boutilier \& Yarusevych 2012c), and does not significantly impact the present investigation since here the interest lies in the relative changes in stability characteristics when the flow is excited.

Figure 11(b) shows a comparison of LST and experimental $N$ factors for $S t=15.6$, where the measured wall-normal velocity fluctuations have been bandpass filtered to within $S t=15.6 \pm 0.2$ in order to compute amplification factors associated with this frequency. A direct comparison of $N$ factors is not possible since disturbances in the experiment can only be detected well downstream of $x_{0}$, where they reach measurable amplitudes. Therefore, following Schmid \& Henningson (2001), the amplification factors are matched at a reference location where the measured disturbance amplitude reaches $A=0.005 U_{0}(x / c=0.44$ in figure $11 b)$, thus allowing for an estimate of $A_{0}$ for a given frequency using equation (3.2). Comparing the LST and experimental $N$ factors reveals that the linear growth of disturbances is accurately captured within $0.42<x / c<0.46$ in the experiment, downstream of which disturbance growth begins to saturate and the agreement with LST deteriorates due to nonlinear effects becoming significant. Similar results are also obtained for both the tonal and broadband excited cases, confirming that LST reliably predicts stability characteristics in the fore portion of the studied separation bubbles.

The changes in stability characteristics with excitation are depicted in figure 12, where contours of the LST predicted spatial growth rates are presented. As per the spatial formulation employed, negative values of $\alpha_{i}$ correspond to convectively amplified disturbances. For the natural case, downstream of separation $\left(x_{S} / c \approx 0.35\right)$, the frequency of the maximum growth rate increases to a value of approximately $S t=13.6$ at $x / c=0.45$, after which the frequency decreases toward the maximum bubble height location $\left(x_{h} / c \approx 0.51\right)$. It is in this region where amplification of disturbances is detected in the experiments and agrees well with the LST predictions (figure 11b). For both types of excitation considered, their application leads to significantly decreased growth rate magnitudes, as the maximum growth rate in the natural flow, $-\alpha_{i} c \approx 52$, decreases by approximately $30 \%$ for both excitation cases (figure $12 b, c)$. A less significant effect is seen on the frequency of maximum growth rates, as both tonal and broadband excitation reduce this frequency to approximately $S t=13.1$ at $x / c=0.42$, i.e. a reduction of approximately $4 \%$. The more significant effect of excitation on maximum growth rates than the corresponding frequency is 


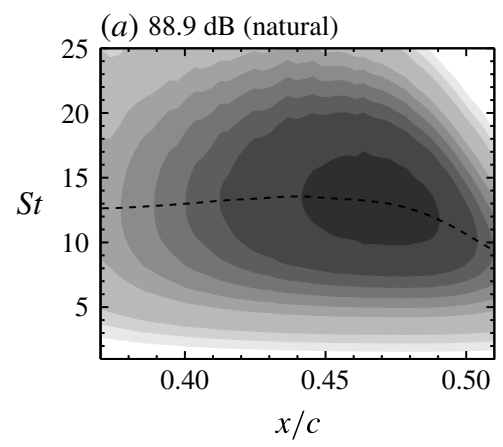

(b) $92.7 \mathrm{~dB}(\mathrm{~T})$

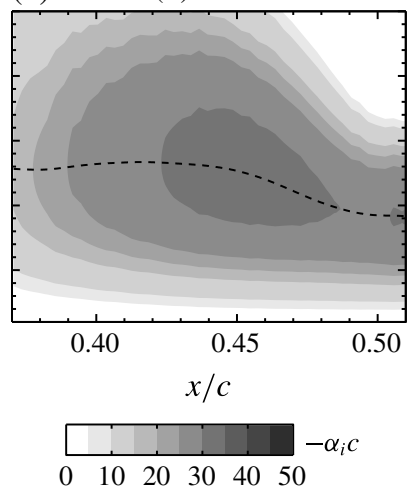

(c) $92.7 \mathrm{~dB}(\mathrm{~B})$

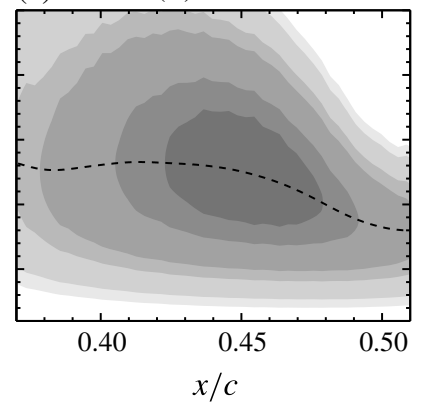

FIGURE 12. Contours of LST predicted spatial growth rates $\left(-\alpha_{i}\right)$. Dashed lines indicate locus of growth rate maxima.

also reported in the works of Marxen \& Henningson (2011), Marxen et al. (2015) and Yarusevych \& Kotsonis (2017b), and is attributed to the mean flow deformation (figure 6). As excitation reduces the size of the separation bubble, the region of instability growth (i.e. the separated shear layer) is brought closer to the wall, which has a damping effect on shear layer disturbances (Dovgal, Kozlov \& Michalke 1994; Diwan \& Ramesh 2009). However, the associated impact on the frequency of the most amplified perturbations is minimal (figure 12).

As established throughout $\$ 3.1$, tonal and broadband excitation at equivalent SPLs (i.e. equivalent input energy levels) produce comparable changes in the mean flow fields (e.g. figures 6 and 9), despite tonal excitation providing a higher initial disturbance amplitude at or close to the frequency at which the LSB is most unstable (figure 12). One hypothesis for this result is that, as seen in figure 12, excitation modifies the frequency of most unstable disturbances, albeit minimally, and so the tonal excitation becomes less effective, while the broadband case is able to excite the new most unstable frequency. This hypothesis is examined in figure 13, where LST predicted $N$ factors and disturbance amplitudes are compared for equivalent tonal and broadband excitation cases.

Consistent with the closely matching spatial growth rates for the considered excitation cases (figure 12b,c), the amplification curves in figure 13(a) show nearly equivalent $N$ factors for frequencies near and below the tonal excitation frequency, $S t=15.6$. Using these LST $N$ factors, the streamwise growth in disturbance amplitude is determined using (3.2) and initial disturbance amplitudes, which are estimated by matching LST and experimental $N$ factors (see discussion of figure $11 b$ ). The resulting LST predicted disturbance amplitudes (figure 13b) show, as expected, the highest initial disturbance amplitude for tonal excitation (cf. figure 4), which, coupled with its $N$ factor curve, results in the tonally excited disturbance outgrowing all disturbances in the broadband case. Thus, according to LST and the theory of transition onset at some critical disturbance amplitude (van Ingen 1956; Smith \& Gamberoni 1956), tonal excitation should lead to earlier transition, which is clearly not the case in the experimental data (figures 6,7 and 9).

It is evident that the assumptions inherent to LST render the technique unable to accurately model the entire transition process. To assess the degree to which disturbance interaction and competition impacts the studied transition processes, the 

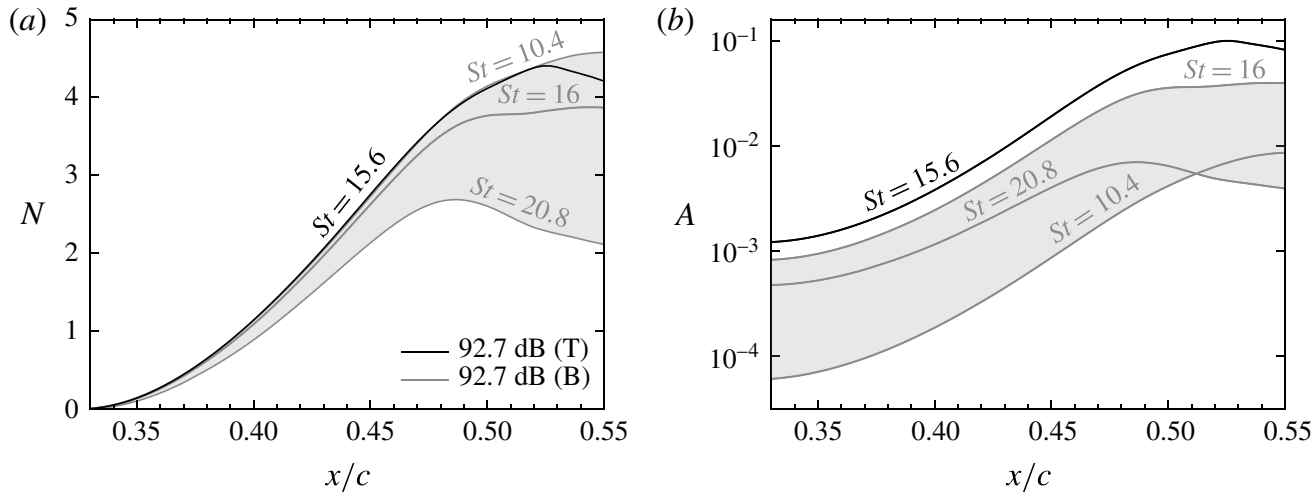

FIGURE 13. Comparison of LST predicted (a) $N$ factors and (b) disturbance amplitudes for frequencies within the excitation bands. Initial disturbance amplitudes are estimated through matching LST and experimental $N$ factors (figure 11b). Curves for all broadband excited frequencies fall within the grey shaded regions.

experimentally measured disturbances are studied via their spectra and spatial growth rates in figure 14. The spectra are computed using Welch's method (Welch 1967) based on 5000 realizations and a final frequency resolution of $S t=0.08$. The growth rates are estimated by integrating the spectra using a band of width $S t= \pm 0.2$ centred at the desired frequency, smoothing the resulting curves using a sliding spatial kernel with width $0.03 c$, and then estimating the local spatial growth rates from the curve slopes. Based on the random error estimates in the PIV measurements, the uncertainty in the spatial growth rates is estimated to be approximately $150 \%$ in regions of weak growth $(x / c<0.5)$, while reducing to approximately $5 \%$ in regions of strong growth $(0.5<x / c<0.55)$.

From figure 14, growth rates for the natural, tonal and broadband cases compare favourably with the LST predictions (figure 12). For the natural case (figure 14a), amplification of disturbances is first detected at approximately $x / c=0.41$ and at the fundamental frequency, followed by disturbances within the unstable frequency band, $10 \lesssim S t \lesssim 20$, amplifying farther downstream. Near the streamwise location of maximum shape factor, there is rapid growth at all measurable frequencies, which is indicative of the onset of transition. A similar progression is seen for the broadband case (figure 14c), except that earlier amplification of disturbances is detected due to the excitation. Most notably, the rapid emergence of growth at all frequencies shifts upstream to approximately $x / c=0.49$, consistent with the location of shape factor maximum. For the case of tonal excitation (figure 14b), a drastic change in the growth of disturbances is observed, as excitation at $S t_{0}=15.6$ effectively confines growth to only that frequency from the beginning of the measured domain to $x / c \approx 0.47$. Growth of disturbances over a wide band of frequencies only begins to occur at $x / c=0.5$, which is where the process takes place for the natural case, despite the drastically different energy input levels (figure 4).

It can be concluded from figure $14(b)$ that the strong amplification of the tonally excited disturbance damps growth of all other disturbances, thus affecting the transition process. This is examined further in figure 15, where experimentally determined perturbation modes for frequencies within the naturally unstable frequency band are presented and compared to LST predicted growth rates. The average uncertainty in mode amplitudes, resulting from the random errors present in the PIV 
(a) $88.9 \mathrm{~dB}$ (natural)
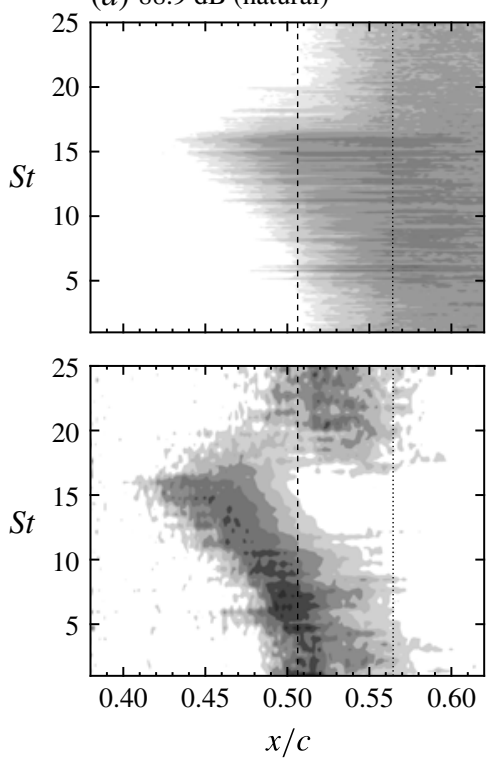

(b) $92.7 \mathrm{~dB}(\mathrm{~T})$
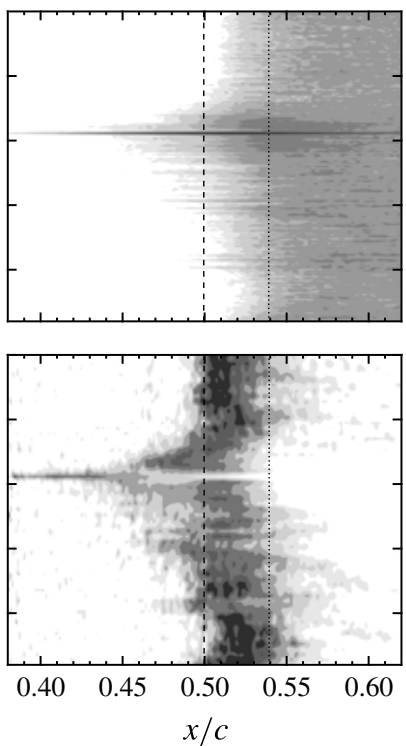

(c) $92.7 \mathrm{~dB}(\mathrm{~B})$
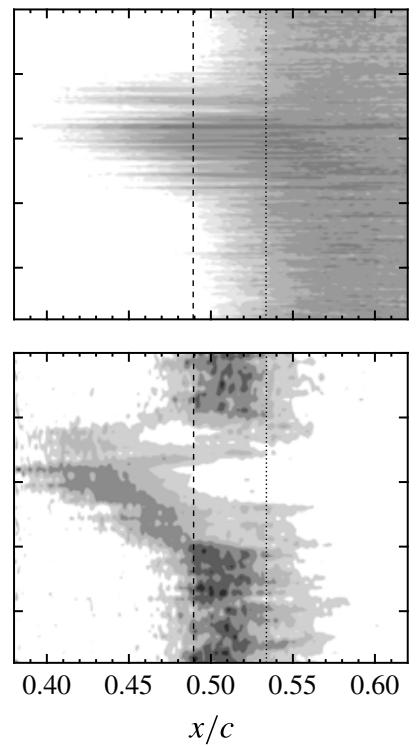

$\square \Phi_{v^{\prime} v^{\prime}}(\mathrm{dB}),-\alpha_{i} c$

$\begin{array}{llllll}0 & 10 & 20 & 30 & 40 & 50\end{array}$

FIGURE 14. Top row: spectra of wall-normal velocity fluctuations $\left(\Phi_{v^{\prime} v^{\prime}}\right)$. Bottom row: spatial growth rates of wall-normal disturbances $\left(-\alpha_{i}\right)$. All quantities are based on velocity measurements within the separated shear layer $\left(y=\delta^{*}\right)$. Dashed and dotted lines denote $x_{H} / c$ and $x_{R} / c$, respectively.

measurements, is estimated be $8 \%$. For the natural case (figure 15a), good agreement is found for frequencies $S t \leqslant 15.6$ in the region where disturbance amplification is first detected, $0.43 \lesssim x / c \lesssim 0.46$. Thus, the growth of these disturbances is independent and is not affected by nonlinear interactions until downstream of $x / c \approx 0.46$. The same can be said for the broadband excited flow (figure 15c), except here agreement with LST is found across all frequencies within the excitation band. The improved agreement with LST at the higher frequencies, $S t>15.6$, is attributed to broadband excitation providing disturbances of significant amplitude at these frequencies for amplification, making it possible to accurately capture the associated velocity fluctuations. When the flow is excited tonally (figure 15b), the damping effect on the growth of disturbances at all frequencies other than that of the excitation becomes immediately apparent, as only the excited mode grows according to its LST predictions, while the growth at all other frequencies is delayed until the excited mode has nearly saturated and nonlinear effects are expected to be significant. Moreover, the agreement between LST predictions and experimental measurements for the tonal excitation frequency persist far downstream, where nonlinear interactions resulted in decreased growth for the natural and broadband excitation cases.

The observed differences in the development of disturbances can be explained through the weakly nonlinear disturbance growth model proposed by Landau (see Landau \& Lifschitz 1987) and further developed by Stuart (1962):

$$
\frac{\mathrm{d}\left|A_{1}\right|^{2}}{\mathrm{~d} x}=2\left|A_{1}\right|^{2}\left(-\alpha_{i}+\sum_{j=1}^{n} \ell_{j}\left|A_{j}\right|^{2}\right),
$$


(a) $88.9 \mathrm{~dB}$ (natural)
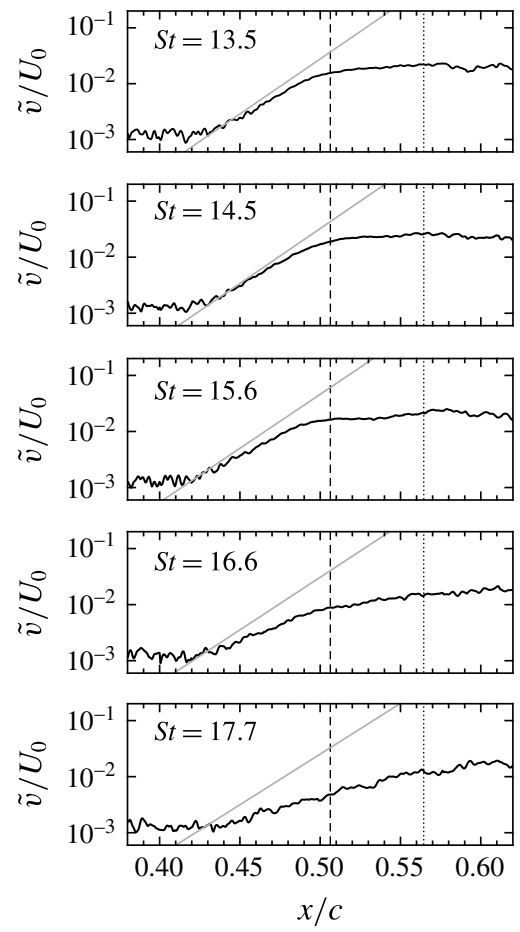

(b) $92.7 \mathrm{~dB}(\mathrm{~T})$
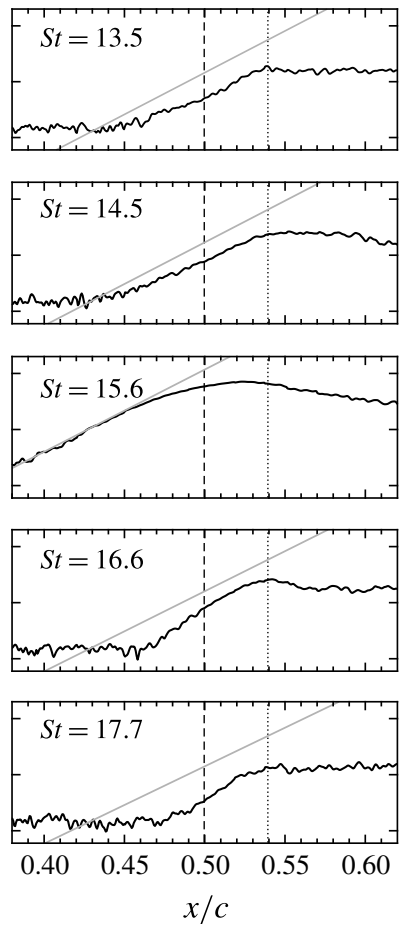

(c) $92.7 \mathrm{~dB}(\mathrm{~B})$
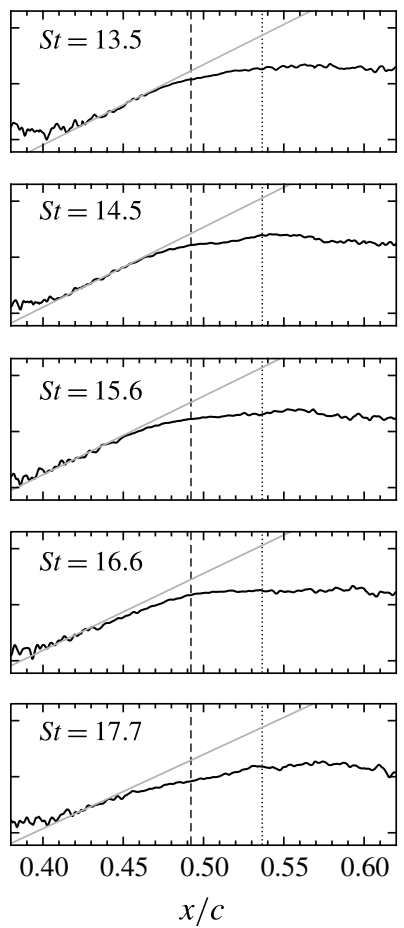

FIGURE 15. Growth of frequency filtered wall-normal disturbances $(\widetilde{v})$ within the separated shear layer $\left(y=\delta^{*}\right)$. Grey lines indicate LST predicted growth rates at $x / c=0.43$. Dashed and dotted lines denote $x_{H} / c$ and $x_{R} / c$, respectively.

which describes the spatial amplification of a disturbance with amplitude $A_{1}$ as a result of its initial linear growth rate, $\alpha_{i}$, and the nonlinear effects imposed by self-interaction $(j=1)$ and interaction with disturbances of all other frequencies $(j \neq 1)$. The Landau coefficients $\left(\ell_{j}\right)$ describe the nature of the interactions, with $\ell_{j}>0$ and $\ell_{j}<0$ corresponding to nonlinear effects resulting in additional amplification or damping, respectively, while linear theory is recovered when $\ell_{j}=0$. Drazin \& Reid (1981) note that the Landau coefficients are generally negative for external flows over bodies, and thus nonlinear effects serve to damp disturbance growth. This is corroborated by the present results, as all instances of good agreement between LST and the experimental measurements are followed by a damping of the experimentally measured disturbances, leading to growth saturation soon after (e.g. figures $11 b$ and 15). Moreover, cross-damping terms have been shown to be much more significant compared to self-damping (Miksad 1973). In addition to the Landau coefficients, equation (3.3) highlights that the degree to which disturbances are damped depends on the amplitude of the disturbance with which the interaction is taking place $\left(A_{j}\right)$. Therefore, the presence of a relatively high amplitude disturbance is expected to damp disturbance growth at all other frequencies. Such is the case observed in figures $14(b)$ and $15(b)$ for the tonal excitation case, while for the broadband case (figures $14 c$ and $15 c$ ) perturbation amplitudes are more moderate, and so all unstable disturbances grow initially at their LST predicted rates, followed by nonlinear damping taking place farther downstream. 
The nonlinear interactions and their impact on transition reported here have, to the authors' knowledge, not been previously reported for separation bubbles. However, similar observations have been reported for boundary layers (Kachanov et al. 1982) and free shear layers (Miksad 1972, 1973). Specifically, Miksad (1973) notes that when exciting free shear layers using two strong acoustic tones, the competing growth of the two instabilities leads to reduced growth rates in comparison to the single excitation case. Furthermore, excitation at multiple frequencies was found to promote the redistribution of fluctuating energy to all possible frequencies, which Miksad linked to a faster increase in the shear layer momentum thickness, and hence an accelerated transition of the shear layer. Similar reports are made by Kachanov et al. (1982), who excited multiple TS modes in a laminar boundary layer. In the present investigation, tonal and broadband excitation lead to strikingly different transition processes but can lead to very similar mean effects on the flow field (figures 6 and 9). It is shown that transition in a laminar separation bubble can be either dominated by a large-amplitude disturbance of a single frequency which damps the growth of all other disturbances (figures $14 b$ and $15 b$ ), or by a band of disturbances of moderate and equal amplitudes, that all initially grow in accordance with linear theory (figure $15 c$ ). For both cases, a rapid redistribution of spectral energy to all frequencies follows (figure 14b,c), with this phenomena occurring earlier for the broadband case, despite equal energy inputs. The 'broadband transition route' is also representative of the natural transition case (figures $14 a$ and $15 a$ ).

Important ramifications regarding the comparison of LST predictions with experimental and numerical results are made clear from the findings of this investigations. Numerous authors have noted this agreement to be surprisingly good (e.g. Marxen, Lang \& Rist 2012; Marxen et al. 2015; Yarusevych \& Kotsonis 2017b), with the valid region extending until very close to where disturbances saturate (Lang et al. 2004), despite the relatively large disturbance amplitudes in this region. Furthermore, and perhaps counter-intuitively, it has been reported that agreement improves with increasing disturbance input levels (Yarusevych \& Kotsonis 2017a). In general, these assertions are supported by the findings presented here (figure 15), with the crucial caveat being that the degree to which LST and experimental/numerical results agree is entirely dictated by the relative importance of nonlinear effects for the particular disturbance mode being considered. For example, when all unstable disturbance amplitudes are small, and thus nonlinear effects are not important, excellent agreement is found with LST until disturbance amplitudes become more moderate (i.e. the broadband excitation case, figure $15 \mathrm{c}$ ). On the other hand, if one disturbance mode is preferentially excited, then its development dominates all others via nonlinear damping, while experiencing strong linear growth of its own (figure $15 b$ ). Thus, it can be conjectured that if only one dominant disturbance mode is present in the flow, then the nonlinear effects imposed by the other, relatively weak, disturbances are not significant and so the dominant mode grows in strong accordance with LST predictions. Miksad (1973) found as much to be true, as agreement between his results and nonlinear theory (3.3) was only found once each Landau coefficient was weighted according to its mode's fractional contribution to the total perturbation energy.

\subsection{Coherent structures}

Thus far, mean features and disturbance development in the separation bubble have been characterized ( $\$ 3.1$ and 3.2, respectively). The link between these two facets of 
(a) $88.9 \mathrm{~dB}$ (natural)

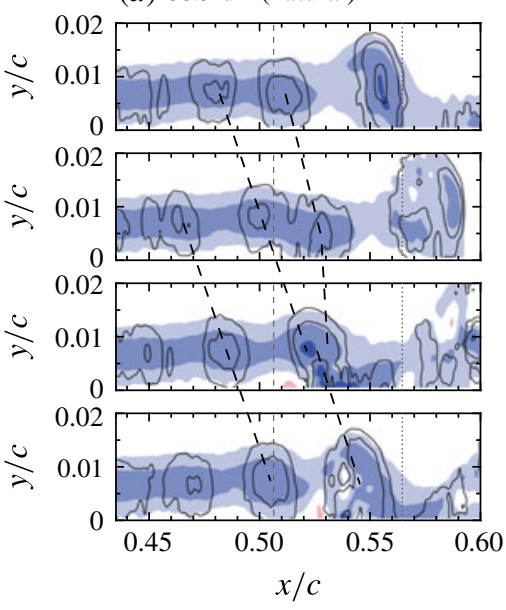

(b) $92.7 \mathrm{~dB}(\mathrm{~T})$

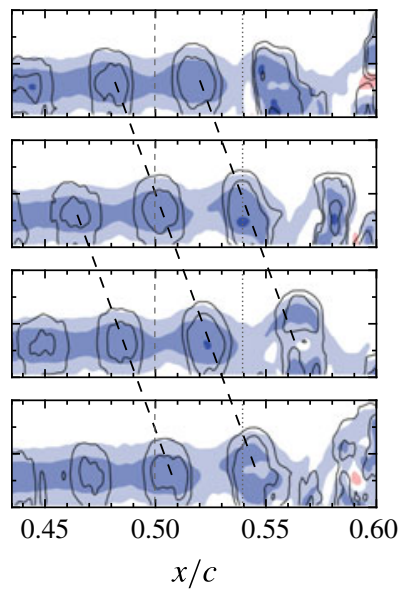

(c) $92.7 \mathrm{~dB}(\mathrm{~B})$

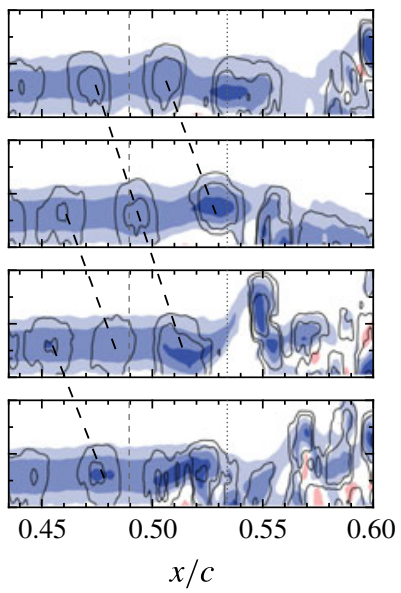

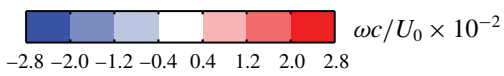

FIGURE 16. (Colour online) Instantaneous contours of spanwise vorticity $(\omega)$. Consecutive frames are separated by $t^{*}=t U_{0} / c=3.8 \times 10^{-2}$. Black lines indicate $\lambda_{2}$-contours (Jeong $\&$ Hussain 1995). Black dashed lines trace the same vortices in a sequence. Grey dashed and dotted lines denote $x_{H} / c$ and $x_{R} / c$, respectively.

the flow is established in this section, as the coherent structures that manifest from the disturbances are examined and their role in producing the observed mean flow field and its statistics is elucidated.

Flow development in the aft portion of the separation bubble for the natural, tonal and broadband cases is depicted in figure 16 using instantaneous contours of spanwise vorticity $(\omega)$. Contours of the $\lambda_{2}$-criterion (Jeong \& Hussain 1995) are added to aid in identifying coherent structures, in addition to dashed lines to assist in tracking individual structures between frames. The spacing and slope of these lines give an indication of the streamwise wavelength and convective velocity of the structures, respectively. Animated sequences are provided as supplementary material (movies 1a-c, available at https://doi.org/10.1017/jfm.2018.546). For all cases, the flow development is characterized by the roll-up of the separated shear layer into vortices upstream of the maximum shape factor location. These shear layer vortices then convect downstream and undergo deformations within the vicinity of mean reattachment, leading to their breakdown to smaller scales. Both the natural and broadband excited flows are quasi-periodic (figure 14a,c), and so significant temporal variability is expected in the flow development. An example of such an occurrence is shown for the natural case (figure 16a), where two vortices develop with sufficiently different convective velocities that they coalesce to form a merged structure. The process may also occur for the broadband excited flow, however, identification of clearly merged structure is difficult due to the earlier onset of breakdown. Vortex merging in naturally developing separation bubbles has also been observed by Kurelek et al. (2016) and Lambert \& Yarusevych (2017).

From figure 16, it is clear that excitation significantly affects flow development, as both tonal and broadband excitation cause vortex formation at earlier streamwise positions, consistent with the upstream shift in $x_{H}$ and earlier detectable disturbance 
amplification (figure 14). Furthermore, by promoting the development of a single harmonic disturbance (figure 15b), it is clear that tonal excitation locks the vortex formation process to the excitation frequency, thus resulting in significantly reduced temporal variability in both the convective velocity and streamwise wavelength of the shed structures. As such, vortex merging is not observed throughout the entire recorded sequence for the presented tonal excitation case. The coherence of the tonally excited structures also appears to be increased in comparison to the broadband case, as the structures in figure $16(b)$ tend to persist farther downstream than those seen in figure 16(c). This has important ramifications regarding mean reattachment, as previous investigators have argued that the shear layer vortices are the primary factor responsible for mean reattachment (Marxen \& Henningson 2011; Yarusevych \& Kotsonis 2017a). This assertion will be examined in detail through the analysis that follows in this section.

The mean convective velocity and streamwise wavelength of the shear layer vortices excited tonally (figure 16b) remain largely unchanged compared to those of the unmerged structures in the natural and broadband excited flow (figure 16a,c), which is attributed to the close matching of the excitation and naturally unstable frequencies. This is verified quantitatively through the computation of two-dimensional, frequency-wavenumber spectra, which are shown in figure 17. The spectra are computed using the wall-normal velocity fluctuations sampled along $y=\delta^{*}$, which are divided into $2^{11} \times 2^{9}$ windows in time and $x$, respectively, with $75 \%$ overlap, resulting in frequency and wavenumber $\left(k_{x}\right)$ resolutions of 0.04 and 20, respectively. For all cases, spectral energy is primarily concentrated along a straight line, which is commonly referred to as the convective ridge (e.g. Howe 1998). Along the convective ridge, the disturbance wavenumber and frequency are related to convective velocity through $U_{c}=2 \pi f / k_{x}$. For the natural case, the non-dimensional convective velocity is $U_{c} / U_{e}=0.56 \pm 0.06$, where the edge velocity is $U_{e}=1.2 U_{0}$ (figure 6). The obtained estimate of $U_{c}$ agrees well with the range $0.3 \lesssim U_{c} / U_{e} \lesssim 0.6$, observed in previous investigations (Burgmann \& Schröder 2008; Boutilier \& Yarusevych 2012c; Pröbsting \& Yarusevych 2015). Along the convective ridge, spectral energy is primarily concentrated within a band centred on $S t=15.6$ and, as expected, overall spectral energy levels are increased when the flow is excited. In the case of tonal excitation (figure 17b), energy is concentrated at the excitation frequency, $S t=15.6$, and the corresponding wavenumber and wavelength on the convective ridge, $k_{x} c=147 \pm 0.5$ and $\lambda_{x} / c=2 \pi / k_{x} c=0.043 \pm 7 \times 10^{-5}$, respectively. This is consistent with the reduction in temporal variability observed in the flow development (figure 16b). For the broadband case (figure 17c), the highest energy levels are associated with the excitation frequency band, $10.4 \leqslant S t \leqslant 20.8$. However, regardless of the excitation type, the slope of the convective ridge remains largely unchanged, with all convective velocities falling within $0.56 \leqslant U_{c} / U_{e} \leqslant 0.58( \pm 0.06)$, which is consistent with the characteristics of the vortices observed in figure 16.

PIV measurements completed in the top-view configuration (figure $3 b$ ) allow for quantitative assessment of both the streamwise and spanwise development of the shear layer vortices. The measurement plane was positioned such that it passed through the top halves of the spanwise rollers, thus allowing for their identification as periodic spanwise bands of high streamwise velocity in the planar fields, as seen in figure 18. Flow is from top to bottom and smoothed spline fits are added to the centre of selected vortices to aid in tracking their development. Animated sequences are provided as supplementary material (movies 2a-c). From figure 18, spanwise coherent structures are first identifiable near the maximum shape factor location, 
(a) $88.9 \mathrm{~dB}$ (natural)

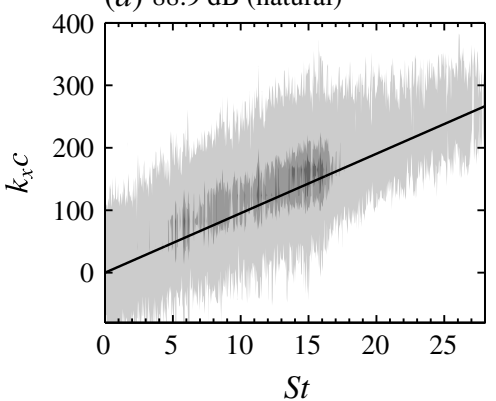

(b) $92.7 \mathrm{~dB}(\mathrm{~T})$

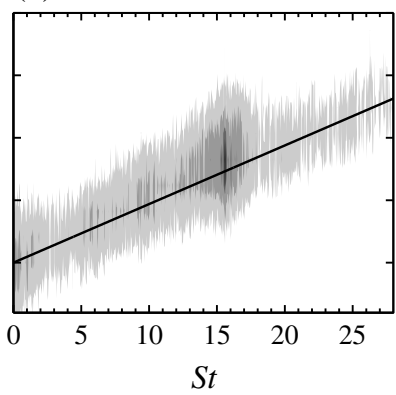

(c) $92.7 \mathrm{~dB}(\mathrm{~B})$

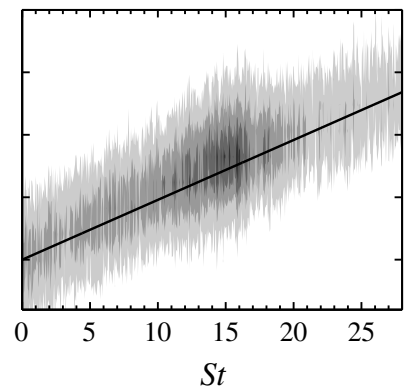

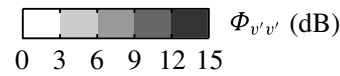

FIGURE 17. Frequency-wavenumber spectra of wall-normal velocity fluctuations $\left(\Phi_{v^{\prime} v^{\prime}}\right)$ within the separated shear layer $\left(y=\delta^{*}\right)$. Solid line is a linear fit estimating the convective ridge.

consistent with where roll-up is observed in the side-view measurements (figure 16). Shortly downstream of roll-up, the structures develop spanwise deformations within the vicinity of the mean reattachment point. These deformations intensify as the structures continue to convect downstream, which eventually leads to the emergence of localized vortex breakup, i.e. the regions of low velocity fluid that appear over the span of the vortex filaments at $x / c \gtrsim 0.55$ throughout figure 18. The formation of spanwise uniform shear layer vortices is consistent with the observations of Jones et al. (2008), Marxen et al. (2013) and Nati et al. (2015), which according to Michelis et al. (2018) is an indication of the relative dominance of normal over oblique modes. Furthermore, the development of spanwise deformations leading to localized regions of vortex breakup is consistent with the vortex breakup mechanism for an LSB proposed by Kurelek et al. (2016).

While general trends in spanwise flow development are similar between the natural and excitation cases, the upstream shift in the roll-up location due to excitation, identified previously in the side-view measurements (figure 16), is also clearly seen in figure 18. Furthermore, both types of excitation appear to modify the predominant spanwise deformation wavelength(s) of the vortex filaments, as a visual comparison between the cases presented in figure 18 suggests that spanwise deformations of shorter wavelengths tend to initially develop when the flow is excited. To affirm this observation, spanwise wavelength characteristics are quantified through wavelet analysis, which is preferred over spatial Fourier analysis due to the limited spanwise extent of the field of view. From the top-view PIV measurements, streamwise fluctuating velocity signals are extracted at several streamwise locations, smoothed using a spatial kernel of width $0.02 c$, and wavelet coefficients are calculated using the Morlet wavelet (Daubechies 1992). Exemplary instantaneous spanwise distributions of velocity fluctuations and their corresponding wavelet coefficients are presented in figure 19. For a given time instant, the predominant spanwise wavelength is estimated from the maximum wavelet coefficient, with the process repeated for all time realizations and statistical samples obtained as a result. The data are presented using histograms in figure 20 at three reference streamwise locations. For the natural case (figure 20a), at $x_{H} / c$ the distribution of $\lambda_{z}$ is nearly symmetric about a mean value of $\overline{\lambda_{z}} / c=0.19$, with predominant wavelengths concentrated within $0.08 \lesssim \lambda_{z} / c \lesssim 0.32$, 


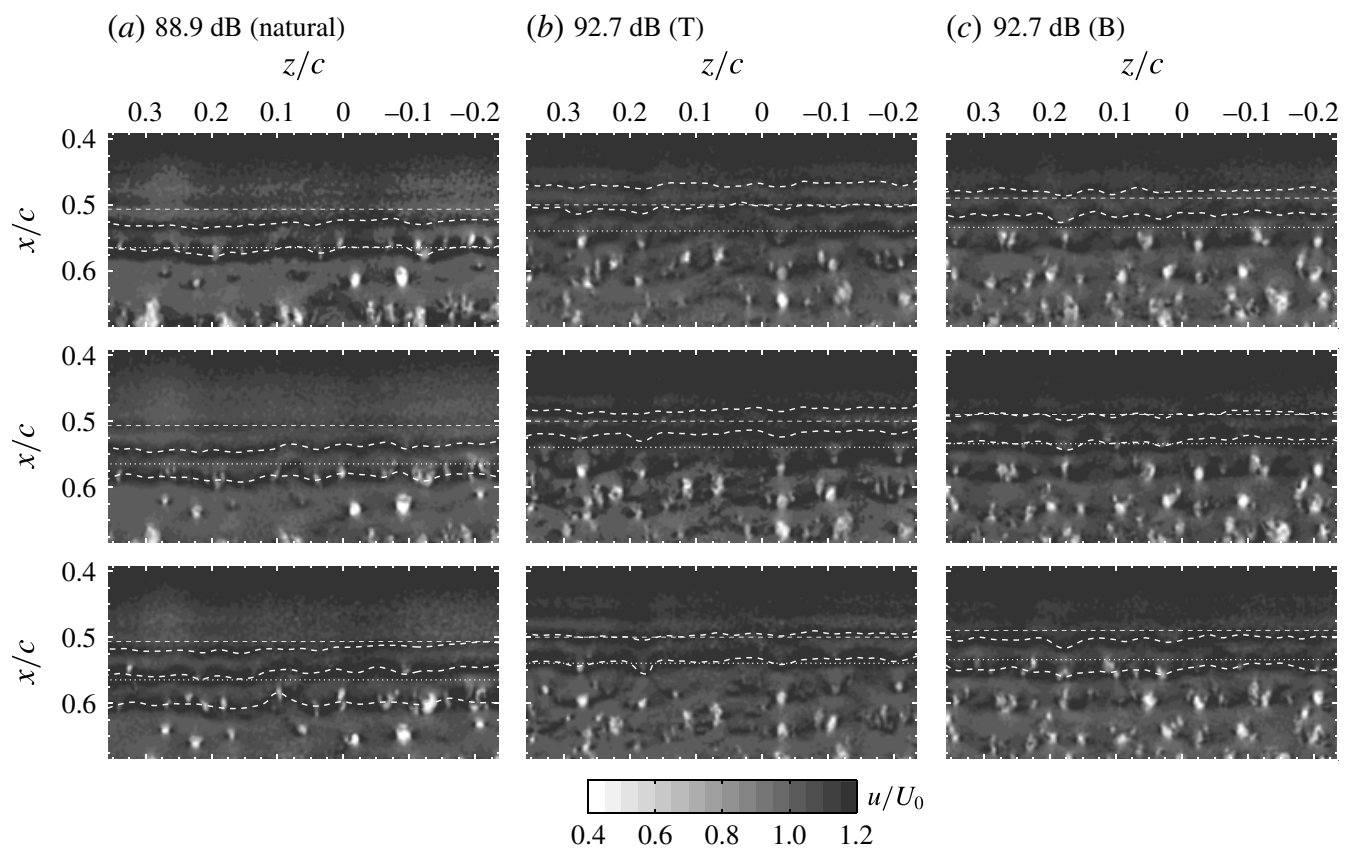

FIGURE 18. Instantaneous contours of streamwise velocity. Consecutive frames are separated by $t^{*}=2.5 \times 10^{-2}$. Thick dashed lines indicate smoothed spline fits to the centre of selected structures. Thin dashed and dotted lines denote $x_{H} / c$ and $x_{R} / c$, respectively.

which is in good agreement with the visualized structures in figure 18. Furthermore, comparing the predominant spanwise and streamwise wavelengths of the structures gives a range of $2 \lesssim \lambda_{z} / \lambda_{x} \lesssim 7$, which is consistent with the results of previous investigations (Marxen et al. 2013; Kurelek et al. 2016; Michelis et al. 2018).

From figure 20(a), the mean spanwise deformation wavelength shifts to lower values as the vortices convect from the maximum shape factor location to the mean reattachment point and beyond it, reaching a value of $\overline{\lambda_{z}} / c=0.11$ at the furthest downstream station. This shift is associated with the aforementioned localized vortex breakup regions seen in figure $18(a)$, with the low velocity zones being separated by a spanwise spacing of about $0.1 c$. In $\$ 3.2$, it was established that disturbances in the broadband excited flow grow in an accelerated, yet similar manner to the natural case, which is also reflected in the development of the spanwise deformations, as figure 20(c) shows that initial deformations occur over a relatively broad range of wavelengths centred at $\lambda_{z} / c \approx 0.2$, which shifts to $\lambda_{z} / c \approx 0.1$ as the vortices convect downstream and begin to break down. In contrast, when the flow is subjected to tonal excitation (figure 20b), at $x_{H} / c$ there is a more pronounced mean tendency in the distribution toward $\lambda_{z} / c=0.1$, indicating that tonal excitation promotes deformations of this wavelength. Thus, tonal excitation is shown to organize shear layer vortex development, not only by locking the shedding frequency and streamwise wavelength (figures $16 b$ and $17 b$ ), but also by reducing variability in spanwise deformations, promoting a spanwise wavelength equal to approximately two times the streamwise wavelength of the structures.

It was stated previously that the coherence of the spanwise vortices is of particular interest since previous investigators have argued these structures are the primary 
(a) $88.9 \mathrm{~dB}$ (natural)
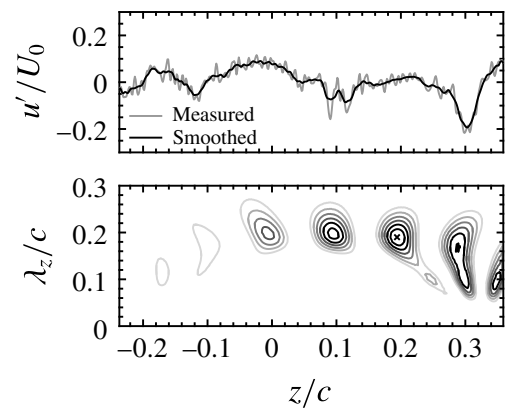

(b) $92.7 \mathrm{~dB}(\mathrm{~T})$
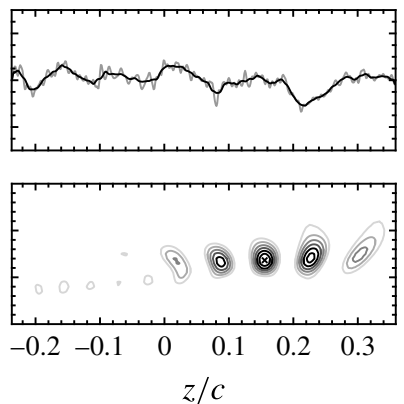

(c) $92.7 \mathrm{~dB}(\mathrm{~B})$
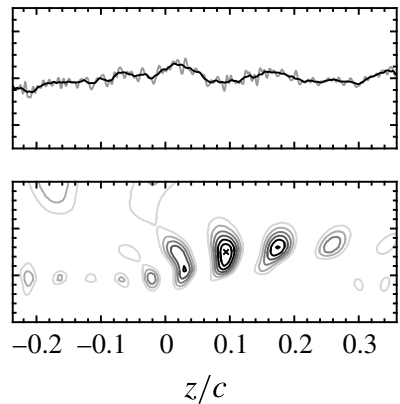

FIGURE 19. Top row: exemplary fluctuating streamwise velocity sampled across the span at $x_{H}$. Bottom row: corresponding wavelet coefficient contours. Maximum wavelet coefficient denoted by $\times$ marker.

(a) $88.9 \mathrm{~dB}$ (natural)
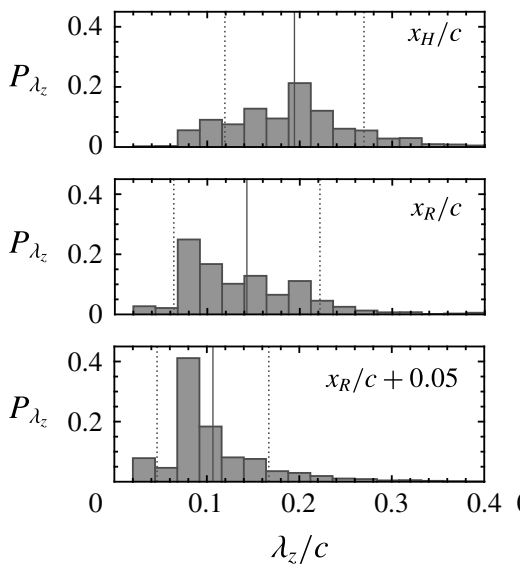

(b) $92.7 \mathrm{~dB}(\mathrm{~T})$
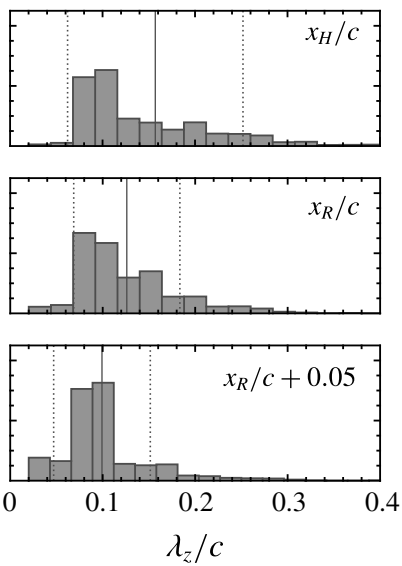

(c) $92.7 \mathrm{~dB}(\mathrm{~B})$
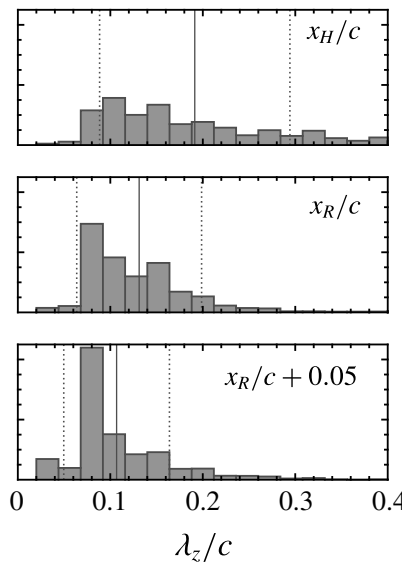

FIGURE 20. Spanwise wavelength probability distributions determined from spatial wavelet analysis (figure 19). Dotted lines indicate standard deviation from the mean (solid line).

factor responsible for mean reattachment (Marxen \& Henningson 2011; Yarusevych \& Kotsonis 2017a), and so their strength should bear some effect on the mean reattachment location for a given set of external flow conditions. This is examined in a quantitative manner by evaluating the spanwise coherence length $\left(l_{z}\right)$ according to:

$$
l_{z}=\int_{0}^{\infty} R_{u u} \mathrm{~d} \Delta z
$$

where $R_{u u}$ is the correlation coefficient function of two $u(t)$ signals measured along a spanwise line at variable spanwise distance $\Delta z$. The difficulty in evaluating $l_{z}$ from experimental data lies in integrating $R_{u u}$ over a finite span when the function must asymptotically tend to zero. To address this, it is common to apply an exponential curve fit to the data (Norberg 2003; Palumbo 2012; Pröbsting et al. 2013):

$$
R_{u u} \approx \exp \left(-\frac{\Delta z}{l_{z}}\right),
$$




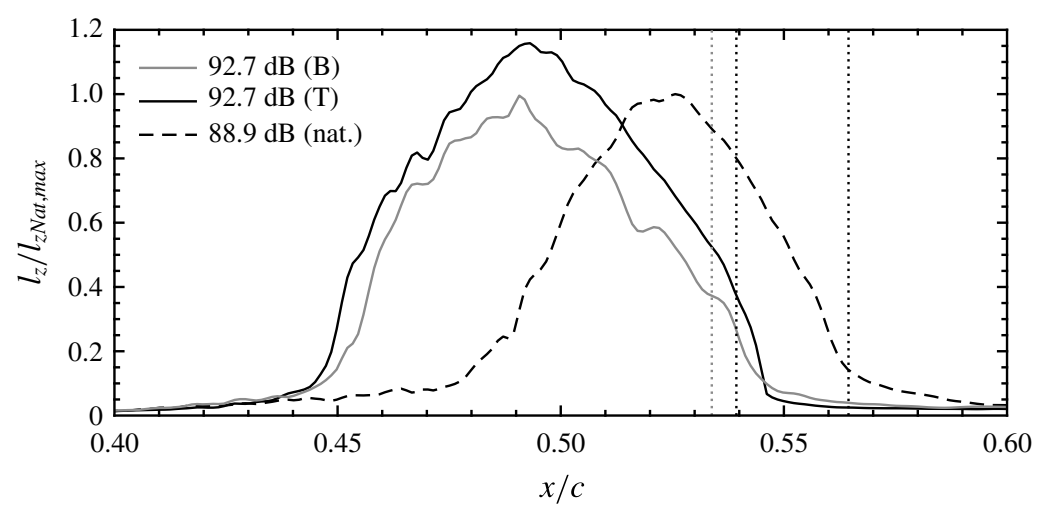

FIGURE 21. Variation of spanwise coherence length with streamwise position. Coherence lengths normalized by maximum value of natural case $\left(l_{\text {zNat,max }}\right)$. Dotted lines (coloured according to legend) denote $x_{R} / c$.

which is convenient since $l_{z}$ arises as the curve fitting coefficient. This approach is employed for the present analysis, with the obtained streamwise variation in coherence length presented in figure 21. For all the cases, a maximum coherence length can be identified, whose streamwise location approximately matches that of the maximum shape factor (cf. figure 9). Upstream of this location, the estimates are subject to progressively higher uncertainty due to relatively low disturbance amplitudes in this region (figures 6 and 15), in addition to increasing deviation between the shear layer core and the position of the laser sheet due to the airfoil surface curvature (figure $3 b$ ). In the fore portion of the bubble, the amplified shear layer disturbances are expected to be strongly two-dimensional (Marxen et al. 2003; Lang et al. 2004), which suggests that the trend upstream of the maxima in $l_{z}$ is a result of disturbance amplitudes progressively increasing above the noise floor. Nevertheless, several key observations can be made from the results. Specifically, both types of excitation lead to equivalent upstream shifts in the streamwise location of the maximum coherence length, consistent with the upstream shift in vortex formation (figure 16) and the changes in mean bubble topology (figures 6 and 9). Moreover, the coherence length for the broadband excitation case peaks at a value that is essentially unchanged compared to the natural case. Thus, broadband excitation does not increase the spanwise coherence of the shear layer vortices, presumably because the excitation does not specifically target any one frequency within the unstable band. In contrast, tonal excitation leads to a marked increase in coherence length, with its peak value being $16 \%$ higher than that of the natural and broadband cases. Following the peak, there is a rapid decrease in coherence for all three cases which is attributed to the rapid development of spanwise deformations and the eventual breakup of the shear layer vortices as they approach and pass the mean reattachment location. For all streamwise locations, the spanwise coherence length for the tonal excitation case exceeds that of the broadband case, confirming that the vortices in the former case maintain stronger coherence farther downstream. However, this does not result in earlier mean reattachment, thus indicating that moderate increases in coherence do not substantially affect the mean reattachment location, while upstream movement in the vortex formation region has significant influence.

To further analyse the effect of excitation on coherent structure characteristics, proper orthogonal decomposition (POD) analysis is performed on the top-view ( $x-z$ 
(a) $88.9 \mathrm{~dB}$ (natural) $z / c$
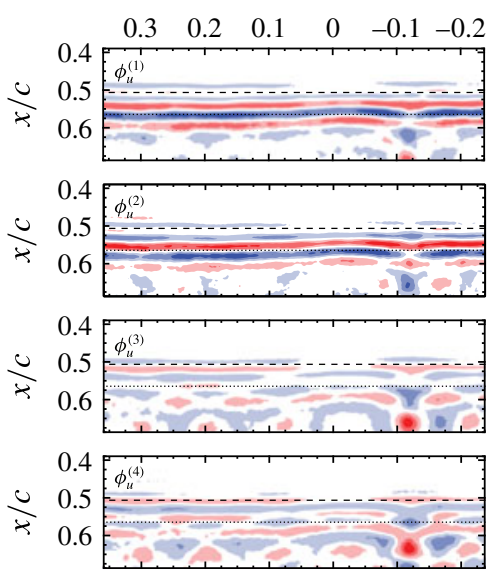

(b) $92.7 \mathrm{~dB}(\mathrm{~T})$ $z / c$
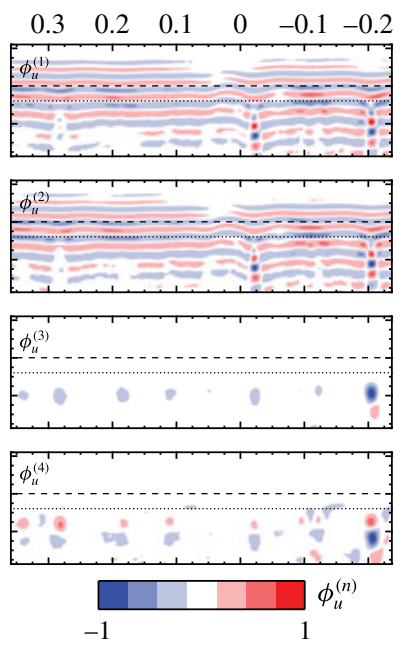

(c) $92.7 \mathrm{~dB}(\mathrm{~B})$
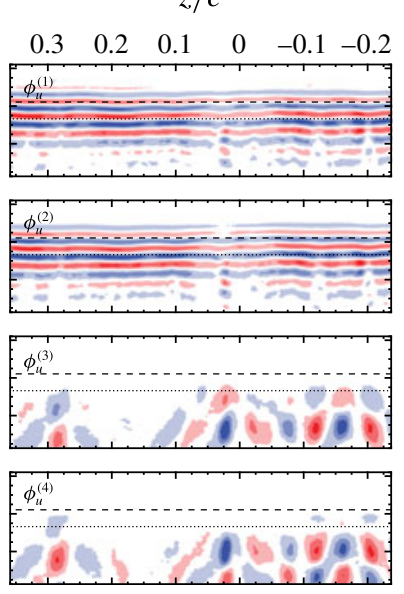

FIGURE 22. (Colour online) Normalized POD spatial modes coloured by the streamwise component $\left(\phi_{u}^{(n)}\right)$. Dashed and dotted lines denote $x_{H} / c$ and $x_{R} / c$, respectively.

plane) measurements using the snapshot method (Sirovich 1987). Figure 22 presents the first four most energetic spatial modes coloured by the streamwise component $\left(\phi_{u}^{(n)}\right)$. From figure 22 it is clear that all modes are paired, i.e. modes 1 and 2 , and modes 3 and 4. A distinct streamwise phase offset of $\pi / 2$ can be seen for each mode pair, which is typical for a number of different flows involving propagating coherent structures (Wee et al. 2004; van Oudheusden et al. 2005; Lengani et al. 2014; Yarusevych \& Kotsonis 2017a). For all cases, the two most energetic modes are associated with the spanwise shear layer vortices, as these modes share several key features with previous results, namely, a consistent streamwise wavelength (cf. figure 16), and spanwise uniform structures that form farther upstream when excited (cf. figure 18). The associated temporal coefficients, not shown for brevity, also feature dominant periodicity at the shedding frequency of the rollers. It is instructive to compare the $\phi_{u}^{(1)}$ and $\phi_{u}^{(2)}$ modes for the tonal and broadband cases in figures 22(b) and 22(c), respectively. Such a comparison reveals that while the structures form at similar streamwise locations, they persist farther downstream in the presence of tonal excitation, thus supporting the earlier observations made from the coherence length estimates (figure 21). This can be further supported through the examination of the relative $\left(E_{r}^{(n)}\right)$ and cumulative $\left(E_{c}^{(n)}\right)$ modal energy distributions, presented in figure 23. Consistent with its excitation input spectrum (figure $4 b$ ), broadband excitation leads to a small increase in the relative energy of the first two modes (figure 23a), and a comparable cumulative distribution over the first twenty modes to the natural case (figure $23 b$ ). In contrast, tonal excitation leads to an increase of approximately $125 \%$ in the most energetic mode pair, which is to be expected given that tonal excitation specifically targets these modes.

Analysis of the top-view measurements revealed that spanwise deformations with a wavelength of $\lambda_{z} / c \approx 0.1$ tend to develop in the vortex filaments in the aft portion of the bubble and downstream of mean reattachment for all cases (figures 18 and 20). Added insight into these deformations is provided by the POD results, as structures 

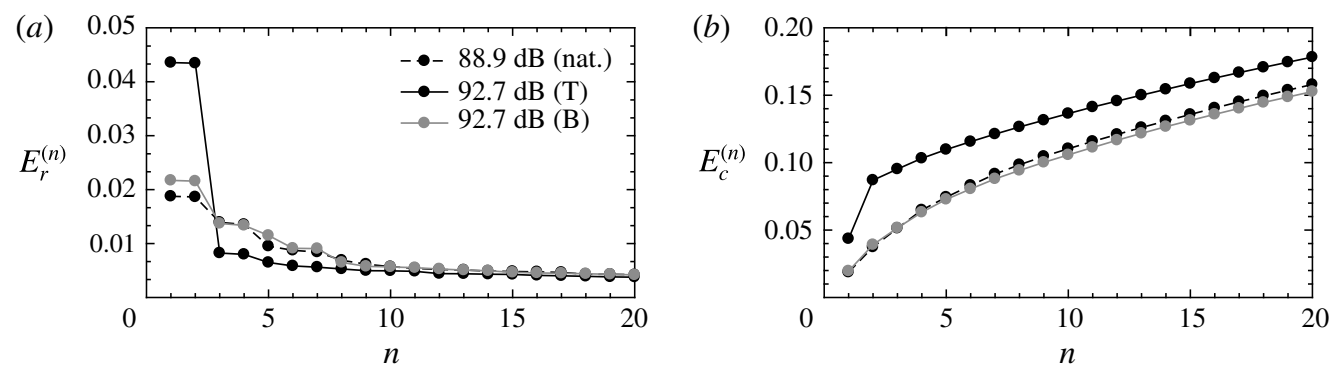

FIgURE 23. Effect of excitation on POD $(a)$ relative and $(b)$ cumulative modal energy distributions.

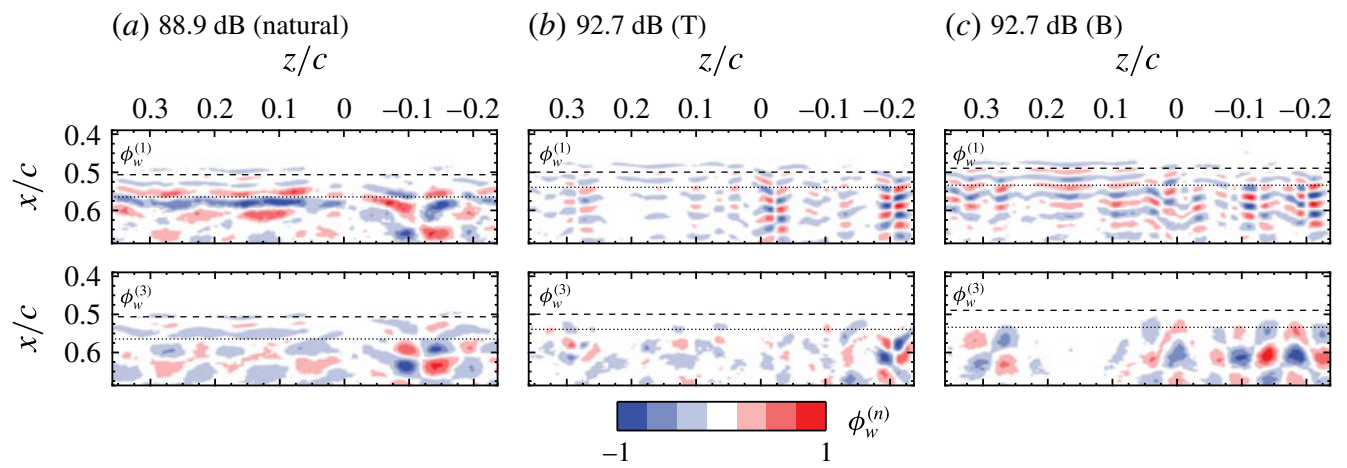

FIgURE 24. (Colour online) Normalized POD spatial modes coloured by the spanwise component $\left(\phi_{w}^{(n)}\right)$. Dashed and dotted lines denote $x_{H} / c$ and $x_{R} / c$, respectively.

with a corresponding spanwise wavelength are evident in the POD spatial modes (e.g. modes 3 and 4 in figure 22). Modes 3 and 4 show temporal periodicity linked to the fundamental frequency, but are associated with a significantly lower energy content compared to modes 1 and 2 (figure 23a). Evidence of prominent spanwise deformations of the main rollers is best captured in the spanwise components of the first four modes, as illustrated in figure 24. This indicates that spanwise deformations tend to occur repeatedly with prevalence at some spanwise locations. A similar observation is made in the experiments of Michelis et al. (2018) (cf. their figure $3 b$ ), who studied a separation bubble formed on a flat plate. In theirs and this experiment, careful attention was paid to the model and facility to prevent any intentional spanwise modulation of disturbances, however, whether the occurrence of these spanwise deformations at these preferred spanwise locations is a result of the underlying physics or is due to some minute imperfection in the experimental set-up is unclear. Nevertheless, it is apparent that such repeated spanwise deformations associated with some preferential wavelength are inherent to the development and breakup of the main shear layers rollers.

\section{Concluding remarks}

The present investigation examines the effects of tonal and broadband acoustic excitation on flow development and transition in a laminar separation bubble. The bubble is formed on the suction side of a NACA 0018 airfoil in a closed-loop 
wind tunnel facility at a Reynolds number of 125000 and an angle of attack of $4^{\circ}$. Disturbances are introduced in a controlled manner from an external source to decouple the interdependence of flow development and acoustic emissions inherent to airfoil self-noise production. The flow field is assessed via time-resolved, two-component PIV. Two separate configurations are employed to evaluate streamwise and spanwise aspects of the flow development.

The results show that, for equivalent energy input levels within the naturally unstable frequency band, tonal and broadband excitation produce similar changes in the mean separation bubble topology. In particular, both result in delayed boundary layer separation, a reduction in the maximum bubble height, and upstream advancement in the shape factor maximum and mean reattachment locations. The location of shape factor maximum is shown to be of particular importance, since it marks the onset of rapid pressure recovery and the beginning of the rapid increase in momentum thickness, providing a robust integral measure for the 'mean transition' location. It is also shown that the velocity fluctuations in the separated shear layer reach comparable critical amplitudes at this location, regardless of excitation type or amplitude.

Local linear stability analysis is shown to accurately model incipient disturbance growth for all cases examined. Consistent with previous investigations (Marxen \& Henningson 2011; Marxen et al. 2015; Yarusevych \& Kotsonis 2017b), the LST results show that excitation significantly reduces disturbance growth rates, while only slightly modifying the frequency of the most amplified disturbances. Despite this modification, according to the LST predictions, the most amplified mode in the presence of tonal excitation should outgrow all individual broadband modes and thus lead to earlier transition for the former case, which is in contradiction with the experimental observations. This is shown to be the result of nonlinear effects. Specifically, in the case of tonal excitation, transition is dominated by the amplification of a single excited wave, which grows in strong accordance with linear theory and significantly damps the growth of all other disturbances as a result of its relatively high amplitude. In contrast, disturbance amplitudes across the entire unstable frequency range are more moderate for the natural and broadband excited flows, and so all unstable disturbances initially grow in accordance with LST. For all cases, a rapid redistribution of perturbation energy to a broad range of frequencies follows, with the phenomenon occurring earliest for the broadband case and at more or less equivalent streamwise locations for the tonal and natural cases, despite drastically different input energy levels.

The significance of nonlinear disturbance interactions reported herein have important ramifications regarding the comparison of LST predictions with experimental and numerical results. Several authors have noted this agreement to be surprisingly good (Lang et al. 2004; Marxen et al. 2012, 2015; Yarusevych \& Kotsonis 2017b) even in flow regions where instability waves attain relatively large amplitudes. In general, these assertions are supported, with the crucial caveat being that agreement is entirely predicated on the relative importance of nonlinear effects for the particular disturbance mode being considered. For example, when all unstable disturbance amplitudes are small and thus nonlinear effects not important, excellent agreement is found with LST until disturbance amplitudes become relatively large, as is observed for the broadband and natural cases. On the other hand, if one disturbance mode is preferentially excited, then its development dominates all others via nonlinear damping. As a consequence, the nonlinear effects imposed on the dominant mode are weak, and so the dominant mode grows in strong accordance with LST. 
Examination of the time-resolved flow development reveals that the unstable flow disturbances produce quasi-periodic shear layer vortices, that form through the roll-up of the separated shear layer just upstream of the maximum shape factor location. For all cases, these structures are strongly two-dimensional and oriented in the spanwise direction at formation, before undergoing significant spanwise deformations, ultimately leading to their breakdown. The spanwise deformations of the shear layer rollers are shown to fall within a range of wavelengths that covers two to seven times their streamwise wavelength. Tonal excitation is shown to have the most distinct effect on the development of the shear layer vortices. Specifically, it leads to earlier formation, a fixed shedding frequency and streamwise wavelength, increased spanwise coherence, and enhances the organization of spanwise deformations at a wavelength equal to two times the streamwise wavelength. In contrast, while advancing the roll-up location due to the increase in the initial amplitude of perturbations, broadband excitation does not appreciably alter any other characteristics compared to the natural case. However, the results suggest that the advancement of shear layer roll-up is the most significant factor influencing separation bubble topology. In particular, for a given angle of attack and Reynolds number, similar separation bubble topologies can be attained with different types of external forcing that induce vortex formation at comparable streamwise locations.

\section{Acknowledgements}

The authors gratefully acknowledge the Natural Sciences and Engineering Research Council of Canada (NSERC) for funding of this work.

\section{Supplementary movies}

Supplementary movies are available at https://doi.org/10.1017/jfm.2018.546.

\section{REFERENCES}

Alam, M. \& SAndham, N. D. 2000 Direct numerical simulation of 'short' laminar separation bubbles with turbulent reattachment. J. Fluid Mech. 403, 223-250.

Amiet, R. K. 1976 Noise due to turbulent flow past a trailing edge. J. Sound Vib. 47 (3), 387-393.

Amitay, M. \& Glezer, A. 2002 Role of actuation frequency in controlled flow reattachment over a stalled airfoil. AIAA J. 40 (2), 209-216.

Arbey, H. \& Bataille, J. 1983 Noise generated by airfoil profiles placed in a uniform laminar flow. J. Fluid Mech. 134, 33-47.

Arcondoulis, E. J. G., Doolan, C. J., Zander, A. C. \& Brooks, L. A. 2010 A review of trailing edge noise generated by airfoils at low to moderate Reynolds number. Acoust. Aust. 38 (3), 129-133.

ATASSI, H. 1984 Feedback in separated flows over symmetric airfoils. In 9th AIAA Aeroacoustics Conference Williamsburg, VA, USA.

Atobe, T., TUinstra, M.\& TAKAgi, S. 2009 Airfoil tonal noise generation in resonant environments. Trans. Japan Soc. Aerosp. Sci. 52 (176), 74-80.

Boiko, A. V., Grek, G. R., Dovgal, A. V. \& Kozlov, V. V. 2002 The Origin of Turbulence in Near-Wall Flows. Springer.

Boutilier, M. S. H. \& YARUSEvych, S. $2012 a$ Effects of end plates and blockage on lowReynolds-number flows over airfoils. AIAA J. 50 (7), 1547-1559.

Boutilier, M. S. H. \& YARUSEVyCh, S. $2012 b$ Parametric study of separation and transition characteristics over an airfoil at low Reynolds numbers. Exp. Fluids 52 (6), 1491-1506. 
Boutilier, M. S. H. \& YARUSEvych, S. 2012c Separated shear layer transition over an airfoil at a low Reynolds number. Phys. Fluids 24 (8), 084105.

Boutilier, M. S. H. \& YARUSEVyCh, S. 2013 Sensitivity of linear stability analysis of measured separated shear layers. Eur. J. Mech. (B/Fluids) 37, 129-142.

Brendel, M. \& Mueller, T. J. 1988 Boundary-layer measurements on an airfoil at low Reynolds numbers. J. Aircraft 25 (7), 612-617.

BRIDGES, T. J. \& MORRIS, P. J. 1984 Differential eigenvalue problems in which the parameter appears nonlinearly. J. Comput. Phys. 55 (3), 437-460.

Brooks, T. F. \& Hodgson, T. H. 1981 Trailing edge noise prediction from measured surface pressures. J. Sound Vib. 78 (1), 69-117.

Brooks, T. F., Pope, D. S. \& Marcolini, M. A. 1989 Airfoil self-noise and prediction. Tech. Rep. NASA Ref. Pub. 1218.

BurgmanN, S. \& SCHRÖDER, W. 2008 Investigation of the vortex induced unsteadiness of a separation bubble via time-resolved and scanning PIV measurements. Exp. Fluids 45 (4), 675-691.

CARmichael, B. H. 1981 Low Reynolds number airfoil survey. NASA Contract Report 165803.

CrAIK, A. D. D. 1971 Non-linear resonant instability in boundary layers. J. Fluid Mech. 50 (02), 393-413.

Daubechies, I. 1992 Ten Lectures on Wavelets. Society for Industrial and Applied Mathematics.

Desquesnes, G., Terracol, M. \& Sagaut, P. 2007 Numerical investigation of the tone noise mechanism over laminar airfoils. J. Fluid Mech. 591, 155-182.

Dini, P., Selig, M. S. \& Maughmer, M. D. 1992 Simplified linear stability transition prediction method for separated boundary layers. AIAA J. 30 (8), 1953-1961.

DiWAN, S. S. \& RAMESH, O. N. 2009 On the origin of the inflectional instability of a laminar separation bubble. J. Fluid Mech. 629, 263-298.

Dovgal, A. V., Kozlov, V. V.\& Michalke, A. 1994 Laminar boundary layer separation: instability and associated phenomena. Prog. Aerosp. Sci. 30, 61-94.

Drazin, P. G. \& ReID, W. H. 1981 Hydrodynamic Stability, 2nd edn. Cambridge University Press.

Fitzgerald, E. J. \& Mueller, T. J. 1990 Measurements in a separation bubble on an airfoil using laser velocimetry. AIAA J. 28 (4), 584-592.

Glezer, A., Amitay, M. \& Honohan, A. M. 2005 Aspects of low- and high-frequency actuation for aerodynamic flow control. AIAA J. 43 (7), 1501-1511.

Häggmark, C. P., Hildings, C. \& Henningson, D. S. 2001 A numerical and experimental study of a transitional separation bubble. Aerosp. Sci. Technol. 5 (5), 317-328.

HAin, R., KÄHLER, C. J. \& RAdespiel, R. 2009 Dynamics of laminar separation bubbles at low-Reynolds-number aerofoils. J. Fluid Mech. 630, 129-153.

Herbert, T. 1988 Secondary instability of boundary layers. Annu. Rev. Fluid Mech. 20, 487-526.

Herbert, T. 1997 Parabolized stability equations. Annu. Rev. Fluid Mech. 29, 245-283.

Hodson, H. P. \& Howell, R. J. 2005 The role of transition in high-lift low-pressure turbines for aeroengines. Prog. Aerosp. Sci. 41 (6), 419-454.

Howe, M. S. 1998 Acoustics of Fluid-Structure Interactions. Cambridge University Press.

VAN INGEN, J. 1956 A suggested semi-empirical method for the calculation of the boundary layer transition region. Tech. Rep. VTH-74. Delft University of Technology.

VAN Ingen, J.\& Kotsonis, M. 2011 A two-parameter method for $e^{N}$ transition prediction. In 6th AIAA Theoretical Fluid Mechanics Conference Honolulu, HI, USA.

Jeong, J. \& Hussain, F. 1995 On the identification of a vortex. J. Fluid Mech. 285, 69-94.

Jones, L. E. \& SANDBERG, R. D. 2011 Numerical analysis of tonal airfoil self-noise and acoustic feedback-loops. J. Sound Vib. 330 (25), 6137-6152.

Jones, L. E., SAndberg, R. D. \& SAndham, N. D. 2010 Stability and receptivity characteristics of a laminar separation bubble on an aerofoil. J. Fluid Mech. 648, 257-296.

Jones, L. E., SANDBerG, R. \& SAndham, N. D. 2008 Direct numerical simulations of forced and unforced separation bubbles on an airfoil at incidence. J. Fluid Mech. 602, 175-207.

Kachanov, Y. S., Kozlov, V. V. \& Levchenko, V. Y. 1982 Origin of turbulence in boundary layers. Novosibirsk: Nauka, (see Boiko et al. 2002). 
Kachanov, Y. S. \& Levchenko, V. Y. 1984 The resonant interaction of disturbances at laminarturbulent transition in a boundary layer. J. Fluid Mech. 138, 209-247.

KirK, T. M. \& YARUSEVyCh, S. 2017 Vortex shedding within laminar separation bubbles forming over an airfoil. Exp. Fluids 58 (5), 43.

Klebanoff, P. S., Tidstrom, K. D. \& Sargent, L. M. 1962 The three-dimensional nature of boundary-layer instability. J. Fluid Mech. 12 (01), 1-34.

Kurelek, J. W., Lambert, A. R. \& Yarusevych, S. 2016 Coherent structures in the transition process of a laminar separation bubble. AIAA J. 54 (8), 2295-2309.

LAMbert, A. R. \& YARUSEVyCH, S. 2017 Characterization of vortex dynamics in a laminar separation bubble. AIAA J. 55 (8), 2664-2675.

Landau, L. D. \& Lifschitz, E. M. 1987 Fluid mechanics. Vol. 6. In Course Theoretical Physics, 2nd edn. Pergamon Press (translated from Russian by J. B. Sykes and W. H. Reid).

LANG, M., RIST, U. \& WAGNER, S. 2004 Investigations on controlled transition development in a laminar separation bubble by means of LDA and PIV. Exp. Fluids 36 (1), 43-52.

Lengani, D., Simoni, D., Ubaldi, M. \& ZUnino, P. 2014 POD analysis of the unsteady behavior of a laminar separation bubble. Exp. Therm. Fluid Sci. 58, 70-79.

Lengani, D., Simoni, D., Ubaldi, M., Zunino, P. \& Bertini, F. 2017 Analysis of the Reynolds stress component production in a laminar separation bubble. Intl J. Heat Fluid Flow 64, $112-119$.

Mankbadi, R. R. 1991 Multifrequency excited jets. Phys. Fluids A 3 (4), 595-605.

MARXEN, O. \& Henningson, D. S. 2011 The effect of small-amplitude convective disturbances on the size and bursting of a laminar separation bubble. J. Fluid Mech. 671, 1-33.

Marxen, O., Kotapati, R. B., Mittal, R. \& Zaki, T. 2015 Stability analysis of separated flows subject to control by zero-net-mass-flux jet. Phys. Fluids 27 (2), 024107.

MARXeN, O., LANG, M. \& RIST, U. 2012 Discrete linear local eigenmodes in a separating laminar boundary layer. J. Fluid Mech. 711, 1-26.

MARXEN, O., LANG, M. \& Rist, U. 2013 Vortex formation and vortex breakup in a laminar separation bubble. J. Fluid Mech. 728, 58-90.

MarXen, O., LANG, M., Rist, U. \& WAGNer, S. 2003 A combined experimental/numerical study of unsteady phenomena in a laminar separation bubble. Flow, Turbul. Combust. 71 (1-4), $133-146$.

MARXEN, O. \& Rist, U. 2010 Mean flow deformation in a laminar separation bubble: separation and stability characteristics. J. Fluid Mech. 660, 37-54.

MCAuliffe, B. R. \& YARAS, M. I. 2005 Separation-bubble-transition measurements on a low-Re airfoil using particle image velocimetry. Vol. 3 Turbo Expo 2005, Parts A B. pp. 1029-1038. ASME.

Melling, A. 1997 Tracer particles and seeding for particle image velocimetry. Meas. Sci. Technol. 8 (12), 1406-1416.

Michelis, T., Kotsonis, M. \& Yarusevych, S. 2018 On the origin of spanwise vortex deformations in laminar separation bubbles. J. Fluid Mech. 841, 81-108.

MiksAD, R. W. 1972 Experiments on the nonlinear stages of free-shear-layer transition. J. Fluid Mech. 56 (04), 695-719.

MiKSAD, R. W. 1973 Experiments on nonlinear interactions in the transition of a free shear layer. J. Fluid Mech. 59 (01), 1-21.

Moffat, R. J. 1988 Describing the uncertainties in experimental results. Exp. Therm. Fluid Sci. 1 (1), 3-17.

Mueller, T. J. \& Delaurier, J. D. 2003 Aerodynamics of small vehicles. Annu. Rev. Fluid Mech. 35 (1), 89-111.

Nash, E. C., Lowson, M. V. \& McAlpine, A. 1999 Boundary-layer instability noise on aerofoils. J. Fluid Mech. 382, 22-61.

Nati, A., De Kat, R., Scarano, F. \& Van Oudheusden, B. W. 2015 Dynamic pitching effect on a laminar separation bubble. Exp. Fluids 56 (9), 172.

Nishioka, M., Asai, M. \& Yoshida, S. 1990 Control of flow separation by acoustic excitation. AIAA J. 28 (11), 1909-1915. 
NorberG, C. 2003 Fluctuating lift on a circular cylinder: review and new measurements. J. Fluids Struct. 17 (1), 57-96.

Ol, M. V., McCauliffe, B. R., Hanff, E. S., Scholz, U. \& Kähler, C. 2005 Comparison of laminar separation bubble measurements on a low Reynolds number airfoil in three facilities. In 35th AIAA Fluid Dynamics Conference Exhibit, Toronto, ON, Canada.

O’Meara, M. \& Mueller, T. J. 1987 Laminar separation bubble characteristics on an airfoil at low Reynolds numbers. AIAA J. 25 (8), 1033-1041.

van Oudheusden, B. W., Scarano, F., van Hinsberg, N. P. \& Watt, D. W. 2005 Phaseresolved characterization of vortex shedding in the near wake of a square-section cylinder at incidence. Exp. Fluids 39 (1), 86-98.

Palumbo, D. 2012 Determining correlation and coherence lengths in turbulent boundary layer flight data. J. Sound Vib. 331 (16), 3721-3737.

PARKER, R. 1966 Resonance effects in wake shedding from parallel plates: some experimental observations. J. Sound Vib. 4 (1), 62-72.

PARKER, R. 1967 Resonance effects in wake shedding from parallel plates: calculation of resonant frequencies. J. Sound Vib. 5 (2), 330-343.

Paterson, R. W., Vogt, P. G., Fink, M. R. \& Munch, C. L. 1973 Vortex noise of isolated airfoils. J. Aircraft 10 (5), 296-302.

Plogmann, B., Herrig, A. \& WÜRZ, W. 2013 Experimental investigations of a trailing edge noise feedback mechanism on a NACA 0012 airfoil. Exp. Fluids 54 (5), 1480.

Pröbsting, S., Scarano, F., Bernardini, M. \& Pirozzoli, S. 2013 On the estimation of wall pressure coherence using time-resolved tomographic PIV. Exp. Fluids 54 (7), 1567.

Pröbsting, S., Scarano, F. \& Morris, S. C. 2015 Regimes of tonal noise on an airfoil at moderate Reynolds number. J. Fluid Mech. 780, 407-438.

Pröbsting, S., Serpieri, J. \& SCARANo, F. 2014 Experimental investigation of aerofoil tonal noise generation. J. Fluid Mech. 747, 656-687.

Pröbsting, S. \& YARUSEVYCH, S. 2015 Laminar separation bubble development on an airfoil emitting tonal noise. J. Fluid Mech. 780, 167-191.

Raffel, M., Willert, C., Wereley, S. \& Kompenhans, J. 2007 Particle Image Velocimetry: A Practical Guide, 2nd edn. Springer.

RAMAN, G. \& RiCE, E. J. 1991 Axisymmetric jet forced by fundamental and subharmonic tones. AIAA J. 29 (7), 1114-1122.

RIST, U. \& MAUCHER, U. 2002 Investigations of time-growing instabilities in laminar separation bubbles. Eur. J. Mech. (B/Fluids) 21 (5), 495-509.

Rodríguez, D., Gennaro, E. M. \& Juniper, M. P. 2013 The two classes of primary modal instability in laminar separation bubbles. J. Fluid Mech. 734, R4.

Rodríguez, D. \& THEOfiLis, V. 2010 Structural changes of laminar separation bubbles induced by global linear instability. J. Fluid Mech. 655, 280-305.

SARIC, W. S. \& Thomas, A. S. W. 1984 Experiments on the subharmonic route to turbulence in boundary layers. In Turbulence choatic Phenomena in Fluids; Proceedings of the International Symposium, Kyoto, Japan, 1983 (ed. T. Tatsumi), pp. 117-122. Elsevier.

SCARAnO, F. \& Riethmuller, M. L. 2000 Advances in iterative multigrid PIV image processing. Exp. Fluids 29 (7), S051-S060.

Schlichting, H. \& Gersten, K. 2000 Boundary-Layer Theory, 8th edn. Springer.

Schmid, P. J. \& Henningson, D. S. 2001 Stability and transition in shear flows. In Applied Mathematical Sciences, Vol. 142 (ed. J. E. Marsden \& L. Sirovich). Springer.

SERnA, J. \& LÁZARo, B. J. 2014 The final stages of transition and the reattachment region in transitional separation bubbles. Exp. Fluids 55 (4), 1695.

Sirovich, L. 1987 Turbulence and the dynamics of coherent structures. Q. Appl. Maths XLV (3), 561-590.

Smith, A. M. O. \& Gamberoni, N. 1956 Transition, pressure gradient and stability theory. Tech. Rep. Douglas Aircraft Company, Inc. 
StUart, J. T. 1962 Non-linear effects in hydrodynamic stability. In Proceedings of the 10th International Congress of Applied Mechanics (ed. F. Rolla \& W. T. Koiter), pp. 63-97. Elsevier.

TANI, I. 1964 Low-speed flows involving bubble separations. Prog. Aerosp. Sci. 5, 70-103.

WatmufF, J. H. 1999 Evolution of a wave packet into vortex loops in a laminar separation bubble. J. Fluid Mech. 31, 119-169.

Wee, D., Yi, T., Annaswamy, A. \& Ghoniem, A. F. 2004 Self-sustained oscillations and vortex shedding in backward-facing step flows: simulation and linear instability analysis. Phys. Fluids 16 (9), 3361-3373.

WELCH, P. 1967 The use of fast Fourier transform for the estimation of power spectra: a method based on time averaging over short, modified periodograms. IEEE Trans. Audio Electroacoust. 15 (2), 70-73.

Westerweel, J., Elsinga, G. E. \& Adrian, R. J. 2013 Particle image velocimetry for complex and turbulent flows. Annu. Rev. Fluid Mech. 45 (1), 409-436.

Westerweel, J. \& ScArano, F. 2005 Universal outlier detection for PIV data. Exp. Fluids 39 (6), 1096-1100.

WIENEKE, B. 2015 PIV uncertainty quantification from correlation statistics. Meas. Sci. Technol. 26 (7), 074002.

Wolf, E., Kähler, C. J., Troolin, D. R., Kykal, C. \& Lai, W. 2011 Time-resolved volumetric particle tracking velocimetry of large-scale vortex structures from the reattachment region of a laminar separation bubble to the wake. Exp. Fluids 50 (4), 977-988.

Wygnanksi, I. J. \& Petersen, R. A. 1987 Coherent motion in excited free shear flows. AIAA J. 25 (2), 201-213.

Yarusevych, S., Kawall, J. G. \& Sullivan, P. E. 2008 Separated-shear-layer development on an airfoil at low Reynolds numbers. AIAA J. 46 (12), 3060-3069.

YARUSEVYCH, S. \& Kotsonis, M. $2017 a$ Effect of local DBD plasma actuation on transition in a laminar separation bubble. Flow, Turbul. Combust. 98 (1), 195-216.

YARUSEVYCH, S. \& KotSONIS, M. $2017 b$ Steady and transient response of a laminar separation bubble to controlled disturbances. J. Fluid Mech. 813, 955-990.

Yarusevych, S., Sullivan, P. E. \& Kawall, J. G. 2007 Effect of acoustic excitation amplitude on airfoil boundary layer and wake development. AIAA J. 45 (4), 760-771.

Yarusevych, S., Sullivan, P. E. \& Kawall, J. G. 2009 On vortex shedding from an airfoil in low-Reynolds-number flows. J. Fluid Mech. 632, 245-271.

Zaman, K. B. M. Q., BAR-Sever, A. \& MAngalam, S. M. 1987 Effect of acoustic excitation on the flow over a low-Re airfoil. J. Fluid Mech. 182, 127-148. 